Keywords: DWPF, sulfur, SB7a, glass, waste loading

Retention: Permanent

\title{
Sulfate Solubility Limit Verification for DWPF Sludge Batch 7a
}

\author{
A. L. Billings
}

April 2011

Savannah River National Laboratory Savannah River Nuclear Solutions, LLC Aiken, SC 29808

Prepared for the U.S. Department of Energy under contract number DE-AC09-08SR22470.

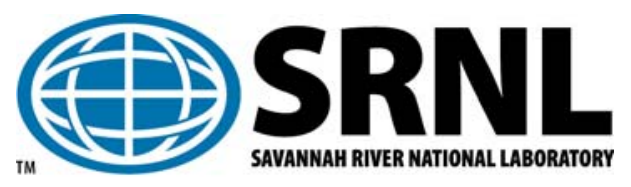




\section{DISCLAIMER}

This work was prepared under an agreement with and funded by the U.S. Government. Neither the U.S. Government or its employees, nor any of its contractors, subcontractors or their employees, makes any express or implied:

1. warranty or assumes any legal liability for the accuracy, completeness, or for the use or results of such use of any information, product, or process disclosed; or

2. representation that such use or results of such use would not infringe privately owned rights; or

3. endorsement or recommendation of any specifically identified commercial product, process, or service.

Any views and opinions of authors expressed in this work do not necessarily state or reflect those of the United States Government, or its contractors, or subcontractors.

\section{Printed in the United States of America \\ Prepared for U.S. Department of Energy}




\section{REVIEWS AND APPROVALS}

AUTHOR:

A. L. Billings, Process Technology Programs

Date

TECHNICAL REVIEW:

K. M. Fox, Process Technology Programs

Date

D. K. Peeler, Process Technology Programs

Date

APPROVAL:

C. C. Herman, Manager

Date

Process Technology Programs

S. L. Marra, Manager

Date

Environmental \& Chemical Process Technology Research Programs

J. E. Occhipinti, Manager

Date

Waste Solidification Engineering 


\section{ACKNOWLEDGEMENTS}

The authors would also like to acknowledge and thank the technical support provided by Pat Simmons and Phyllis Workman for glass fabrication and Dave Best and Whitney Riley for the chemical analysis required for this study. 


\section{EXECUTIVE SUMMARY}

During processing at the Defense Waste Processing Facility (DWPF), high sulfate concentrations in the feed are a concern to DWPF as it can lead to the formation of a detrimental, sulfate-rich, molten salt phase on the surface of the glass melt pool. To avoid these issues, a sulfate concentration limit was implemented into the Product Composition Control System (PCCS). Related to SB7a frit development efforts, the Savannah River National Laboratory (SRNL) assessed the viability of using the current $0.6 \mathrm{wt} \% \mathrm{SO}_{4}{ }^{2-}$ limit set for SB6 (in glass) and the possibility of increasing the $\mathrm{SO}_{4}{ }^{2-}$ solubility limit in PCCS to account for anticipated sulfur concentrations, targeted waste loadings, and inclusion of secondary streams (e.g., Actinide Removal Process (ARP)) with two recommended frits (Frit 418 and Frit 702) for SB7a processing.

For a nominal SB7a blend with a 63 inch SB6 heel remaining in Tank 40 (projection SB7a-63"), a $0.60 \mathrm{wt} \% \mathrm{SO}_{4}{ }^{2-}$ in glass limit was determined for waste loadings of $34 \mathrm{wt} \%$ up to $40 \mathrm{wt} \%$ with Frit 418 based on crucible melts with batched chemicals. SRNL also examined the inclusion of ARP for the same blending scenario (SB7a-63"-ARP) with Frit 418 and at least a $0.6 \mathrm{wt} \% \mathrm{SO}_{4}{ }^{2-}$ level, and waste loadings of $34 \mathrm{wt} \%$ to $40 \mathrm{wt} \%$ were also acceptable. When a visible yellow and/or white sulfate salt layer was visible on the surface of any cooled glass, it was assumed to have surpassed the solubility limit of $\mathrm{SO}_{4}{ }^{2-}$ for that particular composition. All of the glasses fabricated at these concentrations did not exhibit a sulfate rich salt layer on the surface of the glass melt and retained the majority of the batched $\mathrm{SO}_{4}{ }^{2-}$. At higher levels of $\mathrm{SO}_{4}{ }^{2-}$ "spiked" into the projected sludge compositions over the aforementioned interval of waste loadings, with Frit 418 , low viscosity sulfur layers were observed on the surface of glass melts which confirm exceeding the solubility limit. The same sludge scenarios were also tested with Frit 702 and all glasses did not exhibit sulfur layers on the surfaces of the glass melts at spiking levels up to 0.80 wt $\% \mathrm{SO}_{4}{ }^{2-}$. An ultimate $\mathrm{SO}_{4}{ }^{2-}$ limit was not defined with Frit 702 , but if projected $\mathrm{SO}_{4}{ }^{2-}$ concentrations are expected to increase with the onset of SB7a processing, a higher limit is achievable with Frit 702 than is achievable with Frit 418.

Given the anticipated concentration of $\mathrm{SO}_{4}{ }^{2-}$ for $\mathrm{SB} 7 \mathrm{a}$, a $\mathrm{SO}_{4}{ }^{2-}$ limit of 0.6 wt $\% \mathrm{SO}_{4}{ }^{2-}$ is recommended for processing using Frit 418. Once the confirmed SB7a composition is known and should a higher limit be needed, SRNL can re-evaluate the limit based on the actual composition and provide an updated recommendation. It has been observed that higher levels of $\mathrm{SO}_{4}{ }^{2-}$ in glass can be retained with compositional changes to the frit, as was demonstrated by the glasses fabricated using Frit 702. SRNL also recommends the continuation of studies to define a more "global" sulfate concentration limit to account for future sludge batch composition uncertainties. 


\section{TABLE OF CONTENTS}

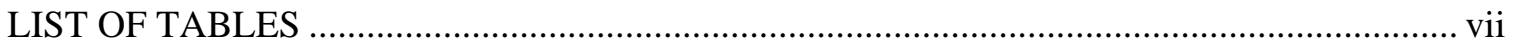

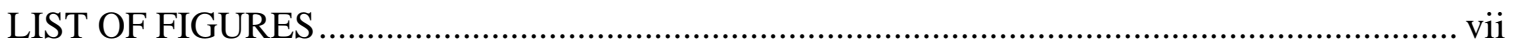

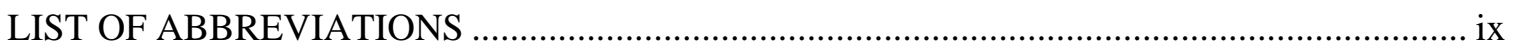

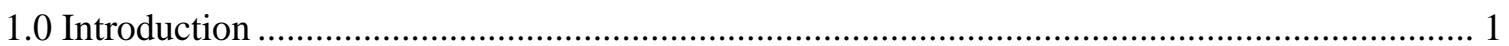

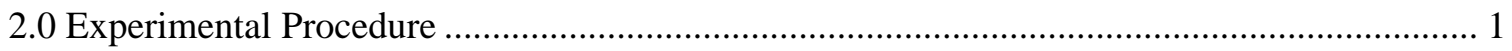

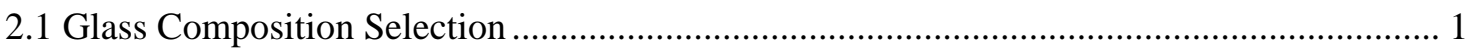

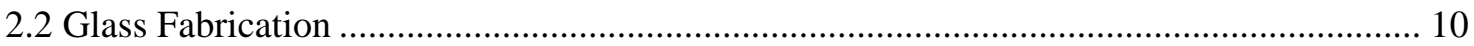

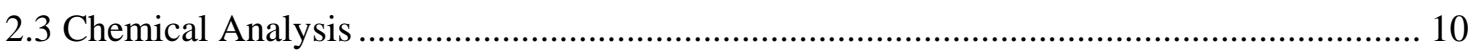

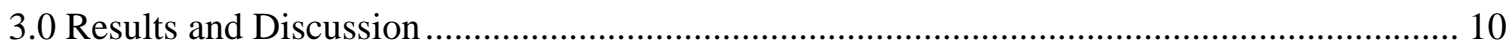

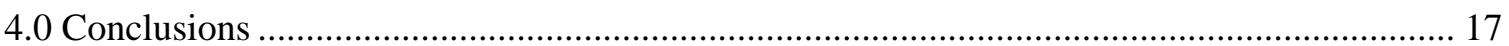

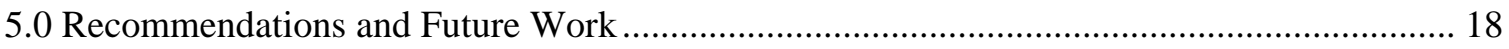

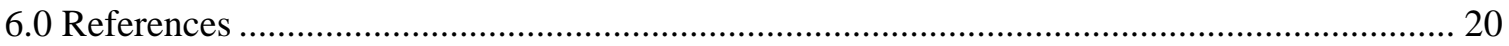




\section{LIST OF TABLES}

Table 2-1. Sludge Compositions Based on the 12-08-10 SB7a Projections from SRR ................. 2

Table 2-2. $\mathrm{SO}_{4}{ }^{2-}$ Limit Required to Reach a Particular Waste Loading ........................................ 3

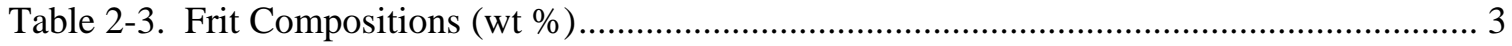

Table 2-4. SB7a Glass Compositions for the Determination of $\mathrm{SO}_{4}{ }^{2-}$ Limit in Glass (SB7a-63”

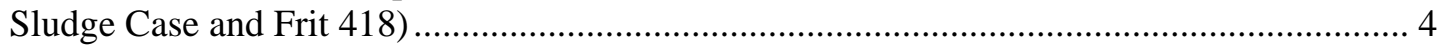

Table 2-5. SB7a Glass Compositions for the Determination of $\mathrm{SO}_{4}{ }^{2-}$ Limit in Glass (SB7a-63”

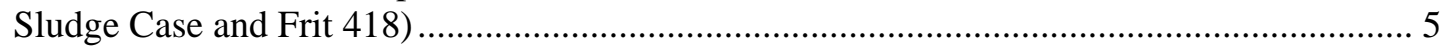

Table 2-6. SB7a Glass Compositions for the Determination of $\mathrm{SO}_{4}{ }^{2-}$ Limit in Glass (SB7a-63”-

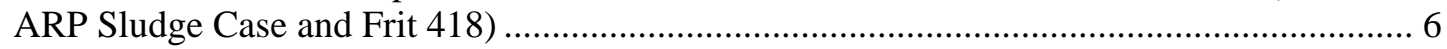

Table 2-7. SB7a Glass Compositions for the Determination of $\mathrm{SO}_{4}{ }^{2-}$ Limit in Glass (SB7a-63”-

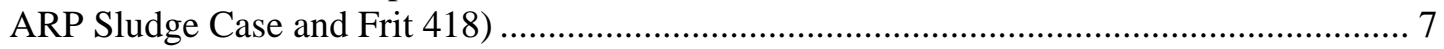

Table 2-8. SB7a Glass Compositions for the Determination of $\mathrm{SO}_{4}{ }^{2-}$ Limit in Glass (SB7a-63”

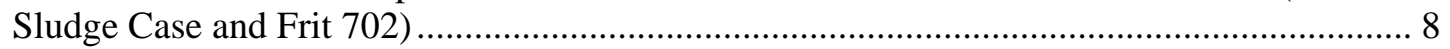

Table 2-9. SB7a Glass Compositions for the Determination of $\mathrm{SO}_{4}{ }^{2-}$ Limit in Glass (SB7a-63"ARP Sludge Case and Frit 702) …............................................................................. 9

Table 3-1. Summary of Visual Observations and Measured $\mathrm{SO}_{4}{ }^{2-}$ for Frit 418 and SB7a-63” with and without ARP at Various $\mathrm{SO}_{4}{ }^{2-}$ Target Concentrations .................................................. 13

Table 3-2. Measured LRM SO $\mathrm{SO}_{3}$ Concentrations for Each Set of Glasses Submitted for

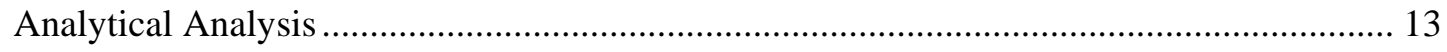

Table 3-3. Summary of Visual Observations and Measured $\mathrm{SO}_{4}{ }^{2-}$ for Frit 702 and SB7a-63” with and without ARP at Various $\mathrm{SO}_{4}{ }^{2-}$ Target Concentrations 16

\section{LIST OF FIGURES}

Figure 1. Visual observations for glasses based on Frit 418 and the SB7a-63" sludge composition as a function of waste loading and targeted $\mathrm{SO}_{4}{ }^{2-}$ content.

Figure 2. Visual observations for glasses based on Frit 418 and the SB7a-63"-ARP sludge composition as a function of waste loading and targeted $\mathrm{SO}_{4}{ }^{2-}$ content. ............................... 12

Figure 3. Visual observations for glasses based on Frit 418 and the SB7a-63” sludge composition as a function of waste loading and measured $\mathrm{SO}_{4}{ }^{2-}$ content.

Figure 4. Visual observations for glasses based on Frit 418 and the SB7a-63"-ARP sludge composition as a function of waste loading and measured $\mathrm{SO}_{4}{ }^{2-}$ content. 14 
Figure 5. Visual observations for glasses based on Frit 702 and the SB7a-63” sludge case and the SB7a-63"-ARP sludge case as a function of waste loading and targeted $\mathrm{SO}_{4}{ }^{2-}$ content........ 15

Figure 6. Visual observations for glasses based on Frit 702 and the SB7a-63" sludge composition as a function of waste loading and measured $\mathrm{SO}_{4}{ }^{2-}$ content................................................. 16

Figure 7. Visual observations for glasses based on Frit 702 and the SB7a-63"-ARP sludge composition as a function of waste loading and measured $\mathrm{SO}_{4}{ }^{2-}$ content. ............................. 17 


\section{LIST OF ABBREVIATIONS}

$\begin{array}{ll}\text { ARP } & \text { Actinide Removal Process } \\ \text { DWPF } & \text { Defense Waste Processing Facility } \\ \text { HLW } & \text { High-Level Waste } \\ \text { ICP-AES } & \text { Inductively Coupled Plasma-Atomic Emission Spectroscopy } \\ \text { LM } & \text { Lithium Metaborate } \\ \text { LRM } & \text { Low-activity test Reference Material } \\ \text { PCCS } & \text { Product Composition Control System } \\ \text { SB } & \text { Sludge Batch } \\ \text { SMRF } & \text { Slurry-fed Melt Rate Furnace } \\ \text { SRNL } & \text { Savannah River National Laboratory } \\ \text { SRR } & \text { Savannah River Remediation } \\ \text { WL } & \text { Waste Loading }\end{array}$




\subsection{Introduction}

During processing at the Defense Waste Processing Facility (DWPF), sulfate from the high-level waste (HLW) feed can partition into at least three paths: (1) incorporate into the glass, (2) volatilize during melting, and (3) result in a separate sulfate-rich salt phase on the surface of the molten glass pool. High sulfate concentration in the feed is a concern to the DWPF as it can lead to the formation of a sulfate-rich, molten salt phase on the surface of the glass melt pool. The presence of this low viscosity melt phase on the surface of the glass melt pool increases corrosion rates of the materials of construction (off-gas system, refractories, and top head components). In addition, there remains the potential for undesirable current paths that could deplete energy delivered to the melter due to the higher electrical conductivity of the molten salt layer. To avoid these issues, a sulfate concentration limit was implemented into the Product Composition Control System (PCCS). For Sludge Batch 1A, 1B, and Sludge Batch 2 (SB1A, SB1B, and SB2), the sulfate solubility limit was $0.4 \mathrm{wt} \% \mathrm{SO}_{4}{ }^{2-}$ in glass. ${ }^{1,2}$ However, because of the increased $\mathrm{SO}_{4}{ }^{2-}$ levels in Sludge Batch 3 (SB3), due to the addition of excess material streams from the canyons and less sludge washing, testing was conducted to determine if the sulfate solubility limit could be increased to minimize washing of SB3. Based on sealed-crucible studies and slurry-fed melt rate furnace (SMRF) runs, the limit was raised to $0.6 \mathrm{wt} \% \mathrm{SO}_{4}{ }^{2-}$ in glass specifically for SB3 with Frit 418. ${ }^{3}$ Sludge Batch 4 (SB4), ${ }^{4}$ Sludge Batch 5 (SB5), ${ }^{5}$ Sludge Batch 6 (SB6), ${ }^{6}$ were also examined on a batch by batch basis, and $0.6 \mathrm{wt} \% \mathrm{SO}_{4}{ }^{2-}$ solubility limit in glass were also recommended. However, the $\mathrm{SO}_{4}{ }^{2-}$ limit set in PCCS remains at $0.4 \mathrm{wt} \% \mathrm{SO}_{4}{ }^{2-}$ in glass and has to be confirmed and set for each new sludge batch. The current task, will examine the next Sludge Batch to be processed at DWPF, Sludge Batch 7a (SB7a) coupled with Frit 418 and Frit 702 in order to set a current $\mathrm{SO}_{4}{ }^{2-}$ solubility limit in glass.

The sulfate retention or solubility of any glass system is a function of its overall composition, which for high-level waste glasses is determined by the sludge composition, frit composition, and waste loading (WL). ${ }^{a}$ Related to SB7a frit development efforts, the Savannah River National Laboratory (SRNL) was to assess the viability of using the current $0.6 \mathrm{wt} \% \mathrm{SO}_{4}{ }^{2-}$ limit (in glass) and the possibility of increasing the $\mathrm{SO}_{4}{ }^{2-}$ solubility limit in PCCS to account for anticipated sulfur concentrations due to uncertainties in the blending strategy, targeted waste loadings, and inclusion of secondary streams (e.g., Actinide Removal Process (ARP)) with two recommended frits (Frit 418 and Frit 702) for SB7a processing. ${ }^{7}$ The Technical Task Request, HLW-DWPFTTR-2010-0014, was written in order to initiate glass formulation and a task to examine the $\mathrm{SO}_{4}{ }^{2-}$ limit specifically for SB7. Work summarized in this report followed the task plan SRNL-RP2010-00908. ${ }^{8}$

\subsection{Experimental Procedure}

\subsection{Glass Composition Selection}

Sludge composition projections for SB7a were provided by Savannah River Remediation (SRR) on December $8,2010^{\mathrm{b}}$ and were used as the basis for formulating glass compositions to determine the $\mathrm{SO}_{4}{ }^{2-}$ limit for SB7a. Table 2-1 lists the as-received sludge projections, on an oxide basis, for a nominal blend scenario with a 63" heel in Tank 40, with and without the ARP ${ }^{9}$ stream. Also, in

\footnotetext{
${ }^{\text {a }}$ Other factors that can influence sulfate retention include processing temperatures, cold cap coverage, feed rate, and melter plenum conditions. These factors can influence the partitioning of sulfate among the glass (retention), off-gas (volatility), and the formation of a salt layer.

${ }^{\mathrm{b}}$ Sludge projections were received via personal communication from Daniel Mcilmoyle to David Peeler and Hasmukh Shah on 12-08-2010 (see SRNL-NB-2010-00108 pp.73-75).
} 
Table 2-1 are the same sludge compositions, renormalized without $\mathrm{U}_{3} \mathrm{O}_{8}$ and $\mathrm{ThO}_{2}$, that were ultimately used for fabricating the glasses for the sulfate solubility study. From this point forward, the renormalized sludge compositions will be referred to as SB7a-63" (sludge only, 63" heel in Tank 40) and SB7a-63”-ARP (SB7a coupled with ARP, 63” heel in Tank 40).

Table 2-1. Sludge Compositions Based on the 12-08-10 SB7a Projections from SRR

\begin{tabular}{|c|c|c|c|c|}
\hline \multirow[b]{2}{*}{ Oxides } & \multicolumn{2}{|c|}{ As-Received } & \multicolumn{2}{|c|}{ Renormalized } \\
\hline & $\begin{array}{c}\text { Sludge Only } \\
\text { TK } 40 \text { Heel 63" } \\
\text { SRR 12-8-10 }\end{array}$ & $\begin{array}{c}\text { With ARP } \\
\text { TK 40 Heel 63" } \\
\text { SRR 12-8-10 }\end{array}$ & $\begin{array}{c}\text { Sludge Only } \\
\text { TK } 40 \text { Heel 63" } \\
\text { SRR 12-8-10 }\end{array}$ & $\begin{array}{c}\text { With ARP } \\
\text { TK } 40 \text { Heel 63" } \\
\text { SRR 12-8-10 }\end{array}$ \\
\hline $\mathbf{A l}_{2} \mathbf{O}_{3}$ & 26.599 & 25.414 & 29.093 & 27.918 \\
\hline $\mathrm{BaO}$ & 0.034 & 0.037 & 0.038 & 0.040 \\
\hline $\mathrm{CaO}$ & 1.237 & 1.217 & 1.353 & 1.337 \\
\hline $\mathrm{Ce}_{2} \mathrm{O}_{3}$ & 0.193 & 0.190 & 0.211 & 0.209 \\
\hline $\mathrm{Cr}_{2} \mathrm{O}_{3}$ & 0.090 & 0.090 & 0.099 & 0.099 \\
\hline $\mathrm{CuO}$ & 0.026 & 0.026 & 0.028 & 0.029 \\
\hline $\mathrm{Fe}_{2} \mathrm{O}_{3}$ & 25.346 & 24.522 & 27.722 & 26.939 \\
\hline $\mathrm{K}_{2} \mathrm{O}$ & 0.012 & 0.017 & 0.014 & 0.019 \\
\hline $\mathbf{L a}_{2} \mathbf{O}_{3}$ & 0.036 & 0.037 & 0.040 & 0.041 \\
\hline MgO & 0.716 & 0.681 & 0.783 & 0.748 \\
\hline MnO & 5.882 & 5.776 & 6.433 & 6.345 \\
\hline $\mathrm{Na}_{2} \mathrm{O}$ & 22.206 & 22.929 & 24.288 & 25.188 \\
\hline $\mathrm{NiO}$ & 3.690 & 3.550 & 4.036 & 3.900 \\
\hline PbO & 0.011 & 0.016 & 0.012 & 0.018 \\
\hline $\mathrm{SO}_{4}{ }^{2-}$ & 1.294 & 1.363 & 1.415 & 1.497 \\
\hline $\mathrm{SiO}_{2}$ & 3.762 & 3.584 & 4.114 & 3.938 \\
\hline $\mathrm{ThO}_{2}$ & 1.030 & 0.972 & -- & -- \\
\hline $\mathrm{TiO}_{2}$ & 0.017 & 1.306 & 0.019 & 1.435 \\
\hline $\mathrm{U}_{3} \mathbf{O}_{8}$ & 7.542 & 7.278 & -- & -- \\
\hline $\mathrm{ZnO}$ & 0.026 & 0.028 & 0.028 & 0.031 \\
\hline $\mathrm{ZrO}_{2}$ & 0.250 & 0.246 & 0.273 & 0.270 \\
\hline
\end{tabular}

Using the sludge projections, a minimum required $\mathrm{SO}_{4}{ }^{2-}$ limit was calculated based upon various waste loadings. That is, if a particular waste loading is targeted, the minimum $\mathrm{SO}_{4}{ }^{2-}$ limit would have to be equal to or greater than the values found in Table 2-2. For example, a targeted 40 wt \% waste loading would require the $\mathrm{SO}_{4}{ }^{2-}$ limit to be equal to or greater than $0.52 \mathrm{wt} \% \mathrm{SO}_{4}{ }^{2-}$ for a sludge-only case, and $0.55 \mathrm{wt} \% \mathrm{SO}_{4}{ }^{2-}$ for coupled operations with ARP on radioactive basis (as-received compositions). This would be equivalent to needing a limit of at least $0.57 \mathrm{wt} \%$ $\mathrm{SO}_{4}{ }^{2-}$ for a sludge-only case, and $0.60 \mathrm{wt} \% \mathrm{SO}_{4}{ }^{2-}$ for coupled operations with ARP on a nonradioactive composition basis (renormalized compositions, or SB7a-63" and SB7a-63"-ARP). Note the renormalized compositions have higher concentrations of $\mathrm{SO}_{4}{ }^{2-}$ due to the removal of the radioactive components $\left(\mathrm{U}_{3} \mathrm{O}_{8}\right.$ and $\left.\mathrm{ThO}_{2}\right)$. This ensures conservatism in setting the limit based upon non-radioactive glasses. 
Table 2-2. $\mathrm{SO}_{4}{ }^{2-}$ Limit Required to Reach a Particular Waste Loading

\begin{tabular}{||c|c|c|c|c||}
\hline WL & $\begin{array}{c}\text { Sludge Only (as- } \\
\text { received) }\end{array}$ & $\begin{array}{c}\text { With ARP (as } \\
\text { received) }\end{array}$ & $\begin{array}{c}\text { Sludge Only } \\
\text { (renormalized, } \\
\text { SB7a-63”) }\end{array}$ & $\begin{array}{c}\text { With ARP } \\
\text { (renormalized, } \\
\text { SB7a-63”-ARP) }\end{array}$ \\
\hline $\mathbf{3 4}$ & 0.44 & 0.46 & 0.48 & 0.51 \\
\hline $\mathbf{3 6}$ & 0.47 & 0.49 & 0.51 & 0.54 \\
\hline $\mathbf{3 8}$ & 0.49 & 0.52 & 0.54 & 0.57 \\
\hline $\mathbf{4 0}$ & 0.52 & 0.55 & 0.57 & 0.60 \\
\hline $\mathbf{4 2}$ & 0.54 & 0.57 & 0.59 & 0.63 \\
\hline
\end{tabular}

Glasses to determine a $\mathrm{SO}_{4}{ }^{2-}$ limit were formulated using the two non-radioactive (renormalized) sludge compositions found in Table 2-1 and two candidate frits, Frit 418 and Frit 702, whose compositions are listed in Table 2-3. Each sludge composition was then "spiked" with enough $\mathrm{SO}_{4}{ }^{2-}$ to target $0.6,0.65,0.7$, and $0.8 \mathrm{wt} \% \mathrm{SO}_{4}{ }^{2-}$ in glass at waste loadings of $34,36,38$, and 40 wt \% after renormalizing to $100 \%$. These sludges were combined with Frit 418 at the aforementioned waste loadings and "spiked" $\mathrm{SO}_{4}{ }^{2-}$ concentrations to form the glass compositions necessary for sulfate testing. The targeted glass compositions that were ultimately fabricated for Frit 418 and the SB7a-63" sludge case are listed in Table 2-4 and Table 2-5. The targeted glass compositions that were ultimately fabricated for Frit 418 and the SB7a-63"-ARP coupled sludge case are listed in Table 2-6 and Table 2-7. The sulfur "spiked", and renormalized sludges were also combined with Frit 702 at the waste loadings of 34, 36, 38, and $40 \mathrm{wt} \%$ at concentrations of 0.7 and $0.8 \mathrm{wt} \% \mathrm{SO}_{4}{ }^{2-}$ in glass in order to evaluate $\mathrm{SO}_{4}{ }^{2-}$ retention with an alternative frit. The targeted glass compositions that were ultimately fabricated for Frit 702 and the SB7a-63" sludge case are listed in Table 2-8 and the targeted compositions for glasses with Frit 702 and the SB7a63"-ARP sludge case are listed in Table 2-9.

Table 2-3. Frit Compositions (wt \%)

\begin{tabular}{|c|c|c|c|c||}
\hline Frit ID & $\mathbf{B}_{2} \mathbf{O}_{3}$ & $\mathbf{L i}_{2} \mathbf{O}$ & $\mathbf{N a}_{2} \mathbf{O}$ & $\mathbf{S i O}_{2}$ \\
\hline $\mathbf{4 1 8}$ & 8 & 8 & 8 & 76 \\
\hline $\mathbf{7 0 2}$ & 8 & 10 & 6 & 76 \\
\hline
\end{tabular}


Table 2-4. SB7a Glass Compositions for the Determination of $\mathrm{SO}_{4}{ }^{2-}$ Limit in Glass (SB7a-63” Sludge Case and Frit 418)

\begin{tabular}{|c|c|c|c|c|c|c|c|c|}
\hline $\begin{array}{c}\text { Glass } \\
\text { ID }\end{array}$ & SL1-01 & SL1-02 & SL1-03 & SL1-04 & SL1-09 & SL1-10 & SL1-11 & SL1-12 \\
\hline Frit & 418 & 418 & 418 & 418 & 418 & 418 & 418 & 418 \\
\hline $\begin{array}{c}\text { Sludge } \\
\text { Type }\end{array}$ & SB7a-63" & SB7a-63" & SB7a-63" & SB7a-63" & SB7a-63" & SB7a-63" & SB7a-63" & SB7a-63" \\
\hline WL & 40 & 40 & 40 & 40 & 38 & 38 & 38 & 38 \\
\hline $\mathrm{Al}_{2} \mathrm{O}_{3}$ & 11.627 & 11.613 & 11.598 & 11.568 & 11.037 & 11.022 & 11.008 & 10.978 \\
\hline $\mathrm{B}_{2} \mathrm{O}_{3}$ & 4.800 & 4.800 & 4.800 & 4.800 & 4.960 & 4.960 & 4.960 & 4.960 \\
\hline $\mathrm{BaO}$ & 0.015 & 0.015 & 0.015 & 0.015 & 0.014 & 0.014 & 0.014 & 0.014 \\
\hline $\mathrm{CaO}$ & 0.541 & 0.540 & 0.540 & 0.538 & 0.513 & 0.513 & 0.512 & 0.511 \\
\hline $\mathrm{Ce}_{2} \mathrm{O}_{3}$ & 0.084 & 0.084 & 0.084 & 0.084 & 0.080 & 0.080 & 0.080 & 0.080 \\
\hline $\mathrm{Cr}_{2} \mathrm{O}_{3}$ & 0.039 & 0.039 & 0.039 & 0.039 & 0.037 & 0.037 & 0.037 & 0.037 \\
\hline $\mathrm{CuO}$ & 0.011 & 0.011 & 0.011 & 0.011 & 0.011 & 0.011 & 0.011 & 0.011 \\
\hline $\mathrm{Fe}_{2} \mathrm{O}_{3}$ & 11.079 & 11.065 & 11.051 & 11.023 & 10.517 & 10.503 & 10.489 & 10.461 \\
\hline $\mathrm{K}_{2} \mathrm{O}$ & 0.005 & 0.005 & 0.005 & 0.005 & 0.005 & 0.005 & 0.005 & 0.005 \\
\hline $\mathrm{La}_{2} \mathrm{O}_{3}$ & 0.016 & 0.016 & 0.016 & 0.016 & 0.015 & 0.015 & 0.015 & 0.015 \\
\hline $\mathrm{Li}_{2} \mathrm{O}$ & 4.800 & 4.800 & 4.800 & 4.800 & 4.960 & 4.960 & 4.960 & 4.960 \\
\hline $\mathrm{MgO}$ & 0.313 & 0.313 & 0.312 & 0.311 & 0.297 & 0.297 & 0.296 & 0.296 \\
\hline $\mathrm{MnO}$ & 2.571 & 2.568 & 2.565 & 2.558 & 2.441 & 2.437 & 2.434 & 2.428 \\
\hline $\mathrm{Na}_{2} \mathrm{O}$ & 14.507 & 14.495 & 14.482 & 14.458 & 14.174 & 14.162 & 14.150 & 14.125 \\
\hline $\mathrm{NiO}$ & 1.613 & 1.611 & 1.609 & 1.605 & 1.531 & 1.529 & 1.527 & 1.523 \\
\hline $\mathrm{PbO}$ & 0.005 & 0.005 & 0.005 & 0.005 & 0.005 & 0.005 & 0.005 & 0.005 \\
\hline $\mathrm{SO}_{4}{ }^{2-}$ & 0.600 & 0.650 & 0.700 & 0.800 & 0.600 & 0.650 & 0.700 & 0.800 \\
\hline $\mathrm{SiO}_{2}$ & 47.244 & 47.242 & 47.240 & 47.236 & 48.681 & 48.679 & 48.677 & 48.673 \\
\hline $\mathrm{TiO}_{2}$ & 0.007 & 0.007 & 0.007 & 0.007 & 0.007 & 0.007 & 0.007 & 0.007 \\
\hline $\mathrm{ZnO}$ & 0.011 & 0.011 & 0.011 & 0.011 & 0.011 & 0.011 & 0.011 & 0.011 \\
\hline $\mathrm{ZrO}_{2}$ & 0.109 & 0.109 & 0.109 & 0.109 & 0.104 & 0.104 & 0.103 & 0.103 \\
\hline
\end{tabular}


Table 2-5. SB7a Glass Compositions for the Determination of $\mathrm{SO}_{4}{ }^{2-}$ Limit in Glass (SB7a-63” Sludge Case and Frit 418)

\begin{tabular}{|c|c|c|c|c|c|c|c|c|}
\hline $\begin{array}{c}\text { Glass } \\
\text { ID } \\
\end{array}$ & SL1-17 & SL1-18 & SL1-19 & SL1-20 & SL1-25 & SL1-26 & SL1-27 & SL1-28 \\
\hline Frit & 418 & 418 & 418 & 418 & 418 & 418 & 418 & 418 \\
\hline $\begin{array}{c}\text { Sludge } \\
\text { Type }\end{array}$ & SB7a-63" & SB7a-63" & SB7a-63" & SB7a-63" & SB7a-63" & SB7a-63" & SB7a-63" & SB7a-63" \\
\hline WL & 36 & 36 & 36 & 36 & 34 & 34 & 34 & 34 \\
\hline $\mathrm{Al}_{2} \mathrm{O}_{3}$ & 10.447 & 10.432 & 10.417 & 10.388 & 9.857 & 9.842 & 9.827 & 9.798 \\
\hline $\mathrm{B}_{2} \mathrm{O}_{3}$ & 5.120 & 5.120 & 5.120 & 5.120 & 5.280 & 5.280 & 5.280 & 5.280 \\
\hline $\mathrm{BaO}$ & 0.014 & 0.014 & 0.013 & 0.013 & 0.013 & 0.013 & 0.013 & 0.013 \\
\hline $\mathrm{CaO}$ & 0.486 & 0.485 & 0.485 & 0.483 & 0.459 & 0.458 & 0.457 & 0.456 \\
\hline $\mathrm{Ce}_{2} \mathrm{O}_{3}$ & 0.076 & 0.076 & 0.075 & 0.075 & 0.071 & 0.071 & 0.071 & 0.071 \\
\hline $\mathrm{Cr}_{2} \mathrm{O}_{3}$ & 0.035 & 0.035 & 0.035 & 0.035 & 0.033 & 0.033 & 0.033 & 0.033 \\
\hline $\mathrm{CuO}$ & 0.010 & 0.010 & 0.010 & 0.010 & 0.010 & 0.010 & 0.010 & 0.009 \\
\hline $\mathrm{Fe}_{2} \mathrm{O}_{3}$ & 9.954 & 9.940 & 9.926 & 9.898 & 9.392 & 9.378 & 9.364 & 9.336 \\
\hline $\mathrm{K}_{2} \mathrm{O}$ & 0.005 & 0.005 & 0.005 & 0.005 & 0.005 & 0.005 & 0.005 & 0.005 \\
\hline $\mathrm{La}_{2} \mathrm{O}_{3}$ & 0.014 & 0.014 & 0.014 & 0.014 & 0.013 & 0.013 & 0.013 & 0.013 \\
\hline $\mathrm{Li}_{2} \mathrm{O}$ & 5.120 & 5.120 & 5.120 & 5.120 & 5.280 & 5.280 & 5.280 & 5.280 \\
\hline $\mathrm{MgO}$ & 0.281 & 0.281 & 0.280 & 0.280 & 0.265 & 0.265 & 0.265 & 0.264 \\
\hline $\mathrm{MnO}$ & 2.310 & 2.307 & 2.304 & 2.297 & 2.180 & 2.176 & 2.173 & 2.167 \\
\hline $\mathrm{Na}_{2} \mathrm{O}$ & 13.841 & 13.829 & 13.817 & 13.792 & 13.509 & 13.496 & 13.484 & 13.459 \\
\hline $\mathrm{NiO}$ & 1.449 & 1.447 & 1.445 & 1.441 & 1.367 & 1.365 & 1.363 & 1.359 \\
\hline $\mathrm{PbO}$ & 0.004 & 0.004 & 0.004 & 0.004 & 0.004 & 0.004 & 0.004 & 0.004 \\
\hline $\mathrm{SO}_{4}{ }^{2-}$ & 0.600 & 0.650 & 0.700 & 0.800 & 0.600 & 0.650 & 0.700 & 0.800 \\
\hline $\mathrm{SiO}_{2}$ & 50.117 & 50.115 & 50.113 & 50.109 & 51.554 & 51.552 & 51.550 & 51.546 \\
\hline $\mathrm{TiO}_{2}$ & 0.007 & 0.007 & 0.007 & 0.007 & 0.006 & 0.006 & 0.006 & 0.006 \\
\hline $\mathrm{ZnO}$ & 0.010 & 0.010 & 0.010 & 0.010 & 0.009 & 0.009 & 0.009 & 0.009 \\
\hline $\mathrm{ZrO}_{2}$ & 0.098 & 0.098 & 0.098 & 0.098 & 0.093 & 0.093 & 0.092 & 0.092 \\
\hline
\end{tabular}


Table 2-6. SB7a Glass Compositions for the Determination of $\mathrm{SO}_{4}{ }^{2-}$ Limit in Glass (SB7a-63”-ARP Sludge Case and Frit 418)

\begin{tabular}{|c|c|c|c|c|c|c|c|c|}
\hline $\begin{array}{c}\text { Glass } \\
\text { ID } \\
\end{array}$ & SL1-05 & SL1-06 & SL1-07 & SL1-08 & SL1-13 & SL1-14 & SL1-15 & SL1-16 \\
\hline Frit & 418 & 418 & 418 & 418 & 418 & 418 & 418 & 418 \\
\hline $\begin{array}{c}\text { Sludge } \\
\text { Type } \\
\end{array}$ & $\begin{array}{c}\text { SB7a-63"- } \\
\text { ARP }\end{array}$ & $\begin{array}{c}\text { SB7a-63"- } \\
\text { ARP }\end{array}$ & $\begin{array}{c}\text { SB7a-63"- } \\
\text { ARP }\end{array}$ & $\begin{array}{c}\text { SB7a-63"- } \\
\text { ARP }\end{array}$ & $\begin{array}{c}\text { SB7a-63"- } \\
\text { ARP }\end{array}$ & $\begin{array}{c}\text { SB7a-63"- } \\
\text { ARP }\end{array}$ & $\begin{array}{c}\text { SB7a-63"- } \\
\text { ARP }\end{array}$ & $\begin{array}{c}\text { SB7a-63"- } \\
\text { ARP }\end{array}$ \\
\hline WL & 40 & 40 & 40 & 40 & 38 & 38 & 38 & 38 \\
\hline $\mathrm{Al}_{2} \mathrm{O}_{3}$ & 11.167 & 11.153 & 11.138 & 11.110 & 10.600 & 10.586 & 10.572 & 10.543 \\
\hline $\mathrm{B}_{2} \mathrm{O}_{3}$ & 4.800 & 4.800 & 4.800 & 4.800 & 4.960 & 4.960 & 4.960 & 4.960 \\
\hline $\mathrm{BaO}$ & 0.016 & 0.016 & 0.016 & 0.016 & 0.015 & 0.015 & 0.015 & 0.015 \\
\hline $\mathrm{CaO}$ & 0.535 & 0.534 & 0.533 & 0.532 & 0.507 & 0.507 & 0.506 & 0.505 \\
\hline $\mathrm{Ce}_{2} \mathrm{O}_{3}$ & 0.083 & 0.083 & 0.083 & 0.083 & 0.079 & 0.079 & 0.079 & 0.079 \\
\hline $\mathrm{Cr}_{2} \mathrm{O}_{3}$ & 0.040 & 0.040 & 0.040 & 0.039 & 0.038 & 0.038 & 0.038 & 0.037 \\
\hline $\mathrm{CuO}$ & 0.012 & 0.012 & 0.012 & 0.012 & 0.011 & 0.011 & 0.011 & 0.011 \\
\hline $\mathrm{Fe}_{2} \mathrm{O}_{3}$ & 10.775 & 10.761 & 10.748 & 10.720 & 10.228 & 10.214 & 10.201 & 10.173 \\
\hline $\mathrm{K}_{2} \mathrm{O}$ & 0.008 & 0.008 & 0.008 & 0.008 & 0.007 & 0.007 & 0.007 & 0.007 \\
\hline $\mathrm{La}_{2} \mathrm{O}_{3}$ & 0.016 & 0.016 & 0.016 & 0.016 & 0.016 & 0.016 & 0.016 & 0.016 \\
\hline $\mathrm{Li}_{2} \mathrm{O}$ & 4.800 & 4.800 & 4.800 & 4.800 & 4.960 & 4.960 & 4.960 & 4.960 \\
\hline $\mathrm{MgO}$ & 0.299 & 0.299 & 0.299 & 0.298 & 0.284 & 0.284 & 0.283 & 0.283 \\
\hline $\mathrm{MnO}$ & 2.538 & 2.535 & 2.532 & 2.525 & 2.409 & 2.406 & 2.403 & 2.396 \\
\hline $\mathrm{Na}_{2} \mathrm{O}$ & 14.875 & 14.862 & 14.849 & 14.824 & 14.524 & 14.511 & 14.498 & 14.472 \\
\hline $\mathrm{NiO}$ & 1.560 & 1.558 & 1.556 & 1.552 & 1.481 & 1.479 & 1.477 & 1.473 \\
\hline $\mathrm{PbO}$ & 0.007 & 0.007 & 0.007 & 0.007 & 0.007 & 0.007 & 0.007 & 0.007 \\
\hline $\mathrm{SO}_{4}{ }^{2-}$ & 0.600 & 0.650 & 0.700 & 0.800 & 0.600 & 0.650 & 0.700 & 0.800 \\
\hline $\mathrm{SiO}_{2}$ & 47.175 & 47.173 & 47.171 & 47.167 & 48.615 & 48.613 & 48.611 & 48.607 \\
\hline $\mathrm{TiO}_{2}$ & 0.574 & 0.573 & 0.572 & 0.571 & 0.545 & 0.544 & 0.543 & 0.542 \\
\hline $\mathrm{ZnO}$ & 0.012 & 0.012 & 0.012 & 0.012 & 0.012 & 0.012 & 0.012 & 0.012 \\
\hline $\mathrm{ZrO}_{2}$ & 0.108 & 0.108 & 0.108 & 0.108 & 0.103 & 0.102 & 0.102 & 0.102 \\
\hline
\end{tabular}


Table 2-7. SB7a Glass Compositions for the Determination of $\mathrm{SO}_{4}{ }^{2-}$ Limit in Glass (SB7a-63”-ARP Sludge Case and Frit 418)

\begin{tabular}{|c|c|c|c|c|c|c|c|c|}
\hline $\begin{array}{c}\text { Glass } \\
\text { ID } \\
\end{array}$ & SL1-21 & SL1-22 & SL1-23 & SL1-24 & SL1-29 & SL1-30 & SL1-31 & SL1-32 \\
\hline Frit & 418 & 418 & 418 & 418 & 418 & 418 & 418 & 418 \\
\hline $\begin{array}{c}\text { Sludge } \\
\text { Type } \\
\end{array}$ & $\begin{array}{c}\text { SB7a-63"- } \\
\text { ARP }\end{array}$ & $\begin{array}{c}\text { SB7a-63"- } \\
\text { ARP }\end{array}$ & $\begin{array}{c}\text { SB7a-63"- } \\
\text { ARP }\end{array}$ & $\begin{array}{c}\text { SB7a-63"- } \\
\text { ARP }\end{array}$ & $\begin{array}{c}\text { SB7a-63"- } \\
\text { ARP }\end{array}$ & $\begin{array}{c}\text { SB7a-63"- } \\
\text { ARP }\end{array}$ & $\begin{array}{c}\text { SB7a-63"- } \\
\text { ARP }\end{array}$ & $\begin{array}{c}\text { SB7a-63"- } \\
\text { ARP }\end{array}$ \\
\hline WL & 36 & 36 & 36 & 36 & 34 & 34 & 34 & 34 \\
\hline $\mathrm{Al}_{2} \mathrm{O}_{3}$ & 10.033 & 10.019 & 10.005 & 9.976 & 9.466 & 9.452 & 9.438 & 9.410 \\
\hline $\mathrm{B}_{2} \mathrm{O}_{3}$ & 5.120 & 5.120 & 5.120 & 5.120 & 5.280 & 5.280 & 5.280 & 5.280 \\
\hline $\mathrm{BaO}$ & 0.014 & 0.014 & 0.014 & 0.014 & 0.014 & 0.014 & 0.014 & 0.014 \\
\hline $\mathrm{CaO}$ & 0.480 & 0.480 & 0.479 & 0.478 & 0.453 & 0.453 & 0.452 & 0.450 \\
\hline $\mathrm{Ce}_{2} \mathrm{O}_{3}$ & 0.075 & 0.075 & 0.075 & 0.075 & 0.071 & 0.071 & 0.071 & 0.070 \\
\hline $\mathrm{Cr}_{2} \mathrm{O}_{3}$ & 0.036 & 0.036 & 0.036 & 0.035 & 0.034 & 0.034 & 0.034 & 0.033 \\
\hline $\mathrm{CuO}$ & 0.010 & 0.010 & 0.010 & 0.010 & 0.010 & 0.010 & 0.010 & 0.010 \\
\hline $\mathrm{Fe}_{2} \mathrm{O}_{3}$ & 9.681 & 9.668 & 9.654 & 9.626 & 9.134 & 9.121 & 9.107 & 9.080 \\
\hline $\mathrm{K}_{2} \mathrm{O}$ & 0.007 & 0.007 & 0.007 & 0.007 & 0.006 & 0.006 & 0.006 & 0.006 \\
\hline $\mathrm{La}_{2} \mathrm{O}_{3}$ & 0.015 & 0.015 & 0.015 & 0.015 & 0.014 & 0.014 & 0.014 & 0.014 \\
\hline $\mathrm{Li}_{2} \mathrm{O}$ & 5.120 & 5.120 & 5.120 & 5.120 & 5.280 & 5.280 & 5.280 & 5.280 \\
\hline $\mathrm{MgO}$ & 0.269 & 0.269 & 0.268 & 0.267 & 0.254 & 0.253 & 0.253 & 0.252 \\
\hline $\mathrm{MnO}$ & 2.280 & 2.277 & 2.274 & 2.268 & 2.152 & 2.148 & 2.145 & 2.139 \\
\hline $\mathrm{Na}_{2} \mathrm{O}$ & 14.172 & 14.159 & 14.147 & 14.121 & 13.821 & 13.808 & 13.795 & 13.770 \\
\hline $\mathrm{NiO}$ & 1.402 & 1.400 & 1.398 & 1.394 & 1.322 & 1.320 & 1.318 & 1.314 \\
\hline $\mathrm{PbO}$ & 0.006 & 0.006 & 0.006 & 0.006 & 0.006 & 0.006 & 0.006 & 0.006 \\
\hline $\mathrm{SO}_{4}{ }^{2-}$ & 0.600 & 0.650 & 0.700 & 0.800 & 0.600 & 0.650 & 0.700 & 0.800 \\
\hline $\mathrm{SiO}_{2}$ & 50.055 & 50.053 & 50.051 & 50.047 & 51.495 & 51.493 & 51.491 & 51.487 \\
\hline $\mathrm{TiO}_{2}$ & 0.516 & 0.515 & 0.514 & 0.513 & 0.486 & 0.486 & 0.485 & 0.484 \\
\hline $\mathrm{ZnO}$ & 0.011 & 0.011 & 0.011 & 0.011 & 0.010 & 0.010 & 0.010 & 0.010 \\
\hline $\mathrm{ZrO}_{2}$ & 0.097 & 0.097 & 0.097 & 0.097 & 0.092 & 0.091 & 0.091 & 0.091 \\
\hline
\end{tabular}


Table 2-8. SB7a Glass Compositions for the Determination of $\mathrm{SO}_{4}{ }^{2-}$ Limit in Glass (SB7a-63” Sludge Case and Frit 702)

\begin{tabular}{|c|c|c|c|c|c|c|c|c|}
\hline $\begin{array}{c}\text { Glass } \\
\text { ID } \\
\end{array}$ & SL2-01 & SL2-02 & SL2-05 & SL2-06 & SL2-09 & SL2-10 & SL2-13 & SL2-14 \\
\hline Frit & 702 & 702 & 702 & 702 & 702 & 702 & 702 & 702 \\
\hline $\begin{array}{c}\text { Sludge } \\
\text { Type }\end{array}$ & SB7a-63" & SB7a-63" & SB7a-63" & SB7a-63" & SB7a-63" & SB7a-63" & SB7a-63" & SB7a-63" \\
\hline WL & 40 & 40 & 38 & 38 & 36 & 36 & 34 & 34 \\
\hline $\mathrm{Al}_{2} \mathrm{O}_{3}$ & 11.598 & 11.568 & 11.008 & 10.978 & 10.417 & 10.388 & 9.827 & 9.798 \\
\hline $\mathrm{B}_{2} \mathrm{O}_{3}$ & 4.800 & 4.800 & 4.960 & 4.960 & 5.120 & 5.120 & 5.280 & 5.280 \\
\hline $\mathrm{BaO}$ & 0.015 & 0.015 & 0.014 & 0.014 & 0.013 & 0.013 & 0.013 & 0.013 \\
\hline $\mathrm{CaO}$ & 0.540 & 0.538 & 0.512 & 0.511 & 0.485 & 0.483 & 0.457 & 0.456 \\
\hline $\mathrm{Ce}_{2} \mathrm{O}_{3}$ & 0.084 & 0.084 & 0.080 & 0.080 & 0.075 & 0.075 & 0.071 & 0.071 \\
\hline $\mathrm{Cr}_{2} \mathrm{O}_{3}$ & 0.039 & 0.039 & 0.037 & 0.037 & 0.035 & 0.035 & 0.033 & 0.033 \\
\hline $\mathrm{CuO}$ & 0.011 & 0.011 & 0.011 & 0.011 & 0.010 & 0.010 & 0.010 & 0.009 \\
\hline $\mathrm{Fe}_{2} \mathrm{O}_{3}$ & 11.051 & 11.023 & 10.489 & 10.461 & 9.926 & 9.898 & 9.364 & 9.336 \\
\hline $\mathrm{K}_{2} \mathrm{O}$ & 0.005 & 0.005 & 0.005 & 0.005 & 0.005 & 0.005 & 0.005 & 0.005 \\
\hline $\mathrm{La}_{2} \mathrm{O}_{3}$ & 0.016 & 0.016 & 0.015 & 0.015 & 0.014 & 0.014 & 0.013 & 0.013 \\
\hline $\mathrm{Li}_{2} \mathrm{O}$ & 6.000 & 6.000 & 6.200 & 6.200 & 6.400 & 6.400 & 6.600 & 6.600 \\
\hline $\mathrm{MgO}$ & 0.312 & 0.311 & 0.296 & 0.296 & 0.280 & 0.280 & 0.265 & 0.264 \\
\hline $\mathrm{MnO}$ & 2.565 & 2.558 & 2.434 & 2.428 & 2.304 & 2.297 & 2.173 & 2.167 \\
\hline $\mathrm{Na}_{2} \mathrm{O}$ & 13.282 & 13.258 & 12.910 & 12.885 & 12.537 & 12.512 & 12.164 & 12.139 \\
\hline $\mathrm{NiO}$ & 1.609 & 1.605 & 1.527 & 1.523 & 1.445 & 1.441 & 1.363 & 1.359 \\
\hline $\mathrm{PbO}$ & 0.005 & 0.005 & 0.005 & 0.005 & 0.004 & 0.004 & 0.004 & 0.004 \\
\hline $\mathrm{SO}_{4}{ }^{2-}$ & 0.700 & 0.800 & 0.700 & 0.800 & 0.700 & 0.800 & 0.700 & 0.800 \\
\hline $\mathrm{SiO}_{2}$ & 47.240 & 47.236 & 48.677 & 48.673 & 50.113 & 50.109 & 51.550 & 51.546 \\
\hline $\mathrm{TiO}_{2}$ & 0.007 & 0.007 & 0.007 & 0.007 & 0.007 & 0.007 & 0.006 & 0.006 \\
\hline $\mathrm{ZnO}$ & 0.011 & 0.011 & 0.011 & 0.011 & 0.010 & 0.010 & 0.009 & 0.009 \\
\hline $\mathrm{ZrO}_{2}$ & 0.109 & 0.109 & 0.103 & 0.103 & 0.098 & 0.098 & 0.092 & 0.092 \\
\hline
\end{tabular}


Table 2-9. SB7a Glass Compositions for the Determination of $\mathrm{SO}_{4}{ }^{2-}$ Limit in Glass (SB7a-63”-ARP Sludge Case and Frit 702)

\begin{tabular}{|c|c|c|c|c|c|c|c|c|}
\hline $\begin{array}{c}\text { Glass } \\
\text { ID } \\
\end{array}$ & SL2-03 & SL2-04 & SL2-07 & SL2-08 & SL2-11 & SL2-12 & SL2-15 & SL2-16 \\
\hline Frit & 702 & 702 & 702 & 702 & 702 & 702 & 702 & 702 \\
\hline $\begin{array}{c}\text { Sludge } \\
\text { Type } \\
\end{array}$ & $\begin{array}{c}\text { SB7a-63"- } \\
\text { ARP }\end{array}$ & $\begin{array}{c}\text { SB7a-63"- } \\
\text { ARP }\end{array}$ & $\begin{array}{c}\text { SB7a-63"- } \\
\text { ARP }\end{array}$ & $\begin{array}{c}\text { SB7a-63"- } \\
\text { ARP }\end{array}$ & $\begin{array}{c}\text { SB7a-63"- } \\
\text { ARP }\end{array}$ & $\begin{array}{c}\text { SB7a-63"- } \\
\text { ARP }\end{array}$ & $\begin{array}{c}\text { SB7a-63"- } \\
\text { ARP }\end{array}$ & $\begin{array}{c}\text { SB7a-63"- } \\
\text { ARP }\end{array}$ \\
\hline WL & 40 & 40 & 38 & 38 & 36 & 36 & 34 & 34 \\
\hline $\mathrm{Al}_{2} \mathrm{O}_{3}$ & 11.138 & 11.110 & 10.572 & 10.543 & 10.005 & 9.976 & 9.438 & 9.410 \\
\hline $\mathrm{B}_{2} \mathrm{O}_{3}$ & 4.800 & 4.800 & 4.960 & 4.960 & 5.120 & 5.120 & 5.280 & 5.280 \\
\hline $\mathrm{BaO}$ & 0.016 & 0.016 & 0.015 & 0.015 & 0.014 & 0.014 & 0.014 & 0.014 \\
\hline $\mathrm{CaO}$ & 0.533 & 0.532 & 0.506 & 0.505 & 0.479 & 0.478 & 0.452 & 0.450 \\
\hline $\mathrm{Ce}_{2} \mathrm{O}_{3}$ & 0.083 & 0.083 & 0.079 & 0.079 & 0.075 & 0.075 & 0.071 & 0.070 \\
\hline $\mathrm{Cr}_{2} \mathrm{O}_{3}$ & 0.040 & 0.039 & 0.038 & 0.037 & 0.036 & 0.035 & 0.034 & 0.033 \\
\hline $\mathrm{CuO}$ & 0.012 & 0.012 & 0.011 & 0.011 & 0.010 & 0.010 & 0.010 & 0.010 \\
\hline $\mathrm{Fe}_{2} \mathrm{O}_{3}$ & 10.748 & 10.720 & 10.201 & 10.173 & 9.654 & 9.626 & 9.107 & 9.080 \\
\hline $\mathrm{K}_{2} \mathrm{O}$ & 0.008 & 0.008 & 0.007 & 0.007 & 0.007 & 0.007 & 0.006 & 0.006 \\
\hline $\mathrm{La}_{2} \mathrm{O}_{3}$ & 0.016 & 0.016 & 0.016 & 0.016 & 0.015 & 0.015 & 0.014 & 0.014 \\
\hline $\mathrm{Li}_{2} \mathrm{O}$ & 6.000 & 6.000 & 6.200 & 6.200 & 6.400 & 6.400 & 6.600 & 6.600 \\
\hline $\mathrm{MgO}$ & 0.299 & 0.298 & 0.283 & 0.283 & 0.268 & 0.267 & 0.253 & 0.252 \\
\hline $\mathrm{MnO}$ & 2.532 & 2.525 & 2.403 & 2.396 & 2.274 & 2.268 & 2.145 & 2.139 \\
\hline $\mathrm{Na}_{2} \mathrm{O}$ & 13.649 & 13.624 & 13.258 & 13.232 & 12.867 & 12.841 & 12.475 & 12.450 \\
\hline $\mathrm{NiO}$ & 1.556 & 1.552 & 1.477 & 1.473 & 1.398 & 1.394 & 1.318 & 1.314 \\
\hline $\mathrm{PbO}$ & 0.007 & 0.007 & 0.007 & 0.007 & 0.006 & 0.006 & 0.006 & 0.006 \\
\hline $\mathrm{SO}_{4}{ }^{2-}$ & 0.700 & 0.800 & 0.700 & 0.800 & 0.700 & 0.800 & 0.700 & 0.800 \\
\hline $\mathrm{SiO}_{2}$ & 47.171 & 47.167 & 48.611 & 48.607 & 50.051 & 50.047 & 51.491 & 51.487 \\
\hline $\mathrm{TiO}_{2}$ & 0.572 & 0.571 & 0.543 & 0.542 & 0.514 & 0.513 & 0.485 & 0.484 \\
\hline $\mathrm{ZnO}$ & 0.012 & 0.012 & 0.012 & 0.012 & 0.011 & 0.011 & 0.010 & 0.010 \\
\hline $\mathrm{ZrO}_{2}$ & 0.108 & 0.108 & 0.102 & 0.102 & 0.097 & 0.097 & 0.091 & 0.091 \\
\hline
\end{tabular}




\subsection{Glass Fabrication}

Glasses were batched and melted following the standard SRNL procedures. ${ }^{10,11}$ The glasses were prepared from the proper proportions of reagent-grade oxides, carbonates, boric acid, and salts in $100 \mathrm{~g}$ batches. The raw materials were thoroughly mixed and placed into $95 \%$ platinum-5\% gold, $300 \mathrm{~mL}$ crucibles and covered with loose fitting platinum-gold lids. The crucibles were placed into an electrically heated, high-temperature furnace at the target melt temperature of $1150{ }^{\circ} \mathrm{C}$. After an isothermal hold at $1150{ }^{\circ} \mathrm{C}$ for 1.0 hour, the crucibles were removed from the furnace and allowed to air cool (quench) while remaining in the crucible. Visual observations were recorded for each glass. Specifically, the glasses in the cooled crucibles were examined for evidence of a yellow sulfur salt layer on the surface of the cooled glass, or the presence of undissolved batch, spinels, large crystals, etc. When a visible yellow and/or white sulfate salt layer was visible on the surface of any cooled glass, it was assumed to have surpassed the solubility limit of $\mathrm{SO}_{4}{ }^{2-}$ for that particular composition. If there was no visible evidence of a sulfur salt layer, that glass composition was concluded to have remained below the sulfur solubility limit. Visual observations regarding $\mathrm{SO}_{4}{ }^{2-}$ retention were confirmed by compositional analysis.

\subsection{Chemical Analysis}

To confirm the quantity of $\mathrm{SO}_{4}{ }^{2-}$ which was retained in each crucible melt, representative samples of each of the glasses fabricated were subjected to chemical analysis. Lithium-metaborate fusion (LM) dissolution was used in order to fully dissolve the glass for measurement of sulfur (S) concentrations. Each glass was prepared in duplicate and all of the prepared samples were analyzed by Inductively Coupled Plasma - Atomic Emission Spectroscopy (ICP-AES) with the instrumentation being re-calibrated between the duplicate analyses. Glass standards were also measured with each submitted set of glasses to assess the performance of the ICP-AES instrument over the course of the analyses. Specifically, several samples of low-activity test reference material (LRM) ${ }^{12}$ were submitted as a check of the precision and accuracy of the ICP-AES analytical technique for measuring sulfur throughout the sample analysis.

\subsection{Results and Discussion}

The highest projected concentration of $\mathrm{SO}_{4}{ }^{2-}$ in glass for the SB7a-63" (non-rad.) sludge case at a $40 \mathrm{wt} \% \mathrm{WL}$ is $0.57 \mathrm{wt} \% \mathrm{SO}_{4}{ }^{2-}$. For coupled operations using the SB7a-63"-ARP (non-rad.) sludge case, a $40 \mathrm{wt} \% \mathrm{WL}$ resulted in a projected $0.60 \mathrm{wt} \% \mathrm{SO}_{4}{ }^{2-}$. These projections remain below and right at the $0.60 \mathrm{wt} \% \mathrm{SO}_{4}{ }^{2-}$ limit set for sludge batches $3,4,5$ and $6 .^{3-6}$ A $0.60 \mathrm{wt} \%$ $\mathrm{SO}_{4}{ }^{2-}$ limit was also expected to be adequate for SB7a, however, knowledge of the point at which the limit would actually be exceeded was considered to be beneficial due to processing uncertainties. The "spiking" method outlined in Section 2.1 allowed for the determination of the $\mathrm{SO}_{4}{ }^{2-}$ solubility limit as a function of WL for each of the sludge cases and frits of interest. Since SB7a has the possibility of processing with and without coupled operations, the $\mathrm{SO}_{4}{ }^{2-}$ limit for SB7a with Frit 418 was determined by the sludge case which exhibited the lowest $\mathrm{SO}_{4}{ }^{2-}$ solubility over the range of waste loadings of interest.

Historically it has been observed that for certain sulfate concentration targets, the presence of sulfate salt layers are highly dependent upon the composition variance imposed by varying waste loadings. ${ }^{3,6}$ For all glasses for the Frit 418, SB7a-63" and SB7a-63”-ARP sludge cases, it was confirmed that no sulfate salt layer formed on the surface of the test melts at $0.6 \mathrm{wt} \% \mathrm{SO}_{4}{ }^{2-}$ up to a waste loading of $40 \%$. The visual results of both sludge cases with Frit 418 as a function of waste loading and targeted $\mathrm{SO}_{4}{ }^{2-}$ content are displayed in Figure 1 and Figure 2. For the Frit 418 
and SB7a-63" sludge case glasses, sulfate layers were observed at spiking concentrations of 0.65 $\mathrm{wt} \% \mathrm{SO}_{4}{ }^{2-}$ at waste loadings of 36 and $38 \mathrm{wt} \%$. At concentrations of $0.7 \mathrm{wt} \% \mathrm{SO}_{4}{ }^{2-}$ and greater at 34, 36 and $38 \mathrm{wt} \% \mathrm{WL}$, sulfate was also observed. A sulfur layer was not observed at $40 \%$ WL until spiking levels of $0.80 \mathrm{wt} \% \mathrm{SO}_{4}{ }^{2-}$ were attempted.

For the Frit 418 and SB7a-63”-ARP sludge case, it appeared the $\mathrm{SO}_{4}{ }^{2-}$ limit was slightly higher than the sludge only system with the same frit (Figure 2). A visible sulfur layer on the surface of the glass was only observed for spiking levels of $0.80 \mathrm{wt} \% \mathrm{SO}_{4}{ }^{2-}$ at waste loadings of 34 and 38 wt \%. This apparent increase in sulfate solubility may be related to the very slight composition differences which occur with the addition of ARP streams to the sludge waste. In glasses fabricated with the SB7a-63"-ARP sludge type, total $\mathrm{Fe}_{2} \mathrm{O}_{3}, \mathrm{Al}_{2} \mathrm{O}_{3}$, and $\mathrm{SiO}_{2}$ decreases and $\mathrm{Na}_{2} \mathrm{O}$ increases. This combination of the major glass formers shifting slightly demonstrates the significance of slight composition shifts and their impact on sulfate solubility for these glass composition regions.

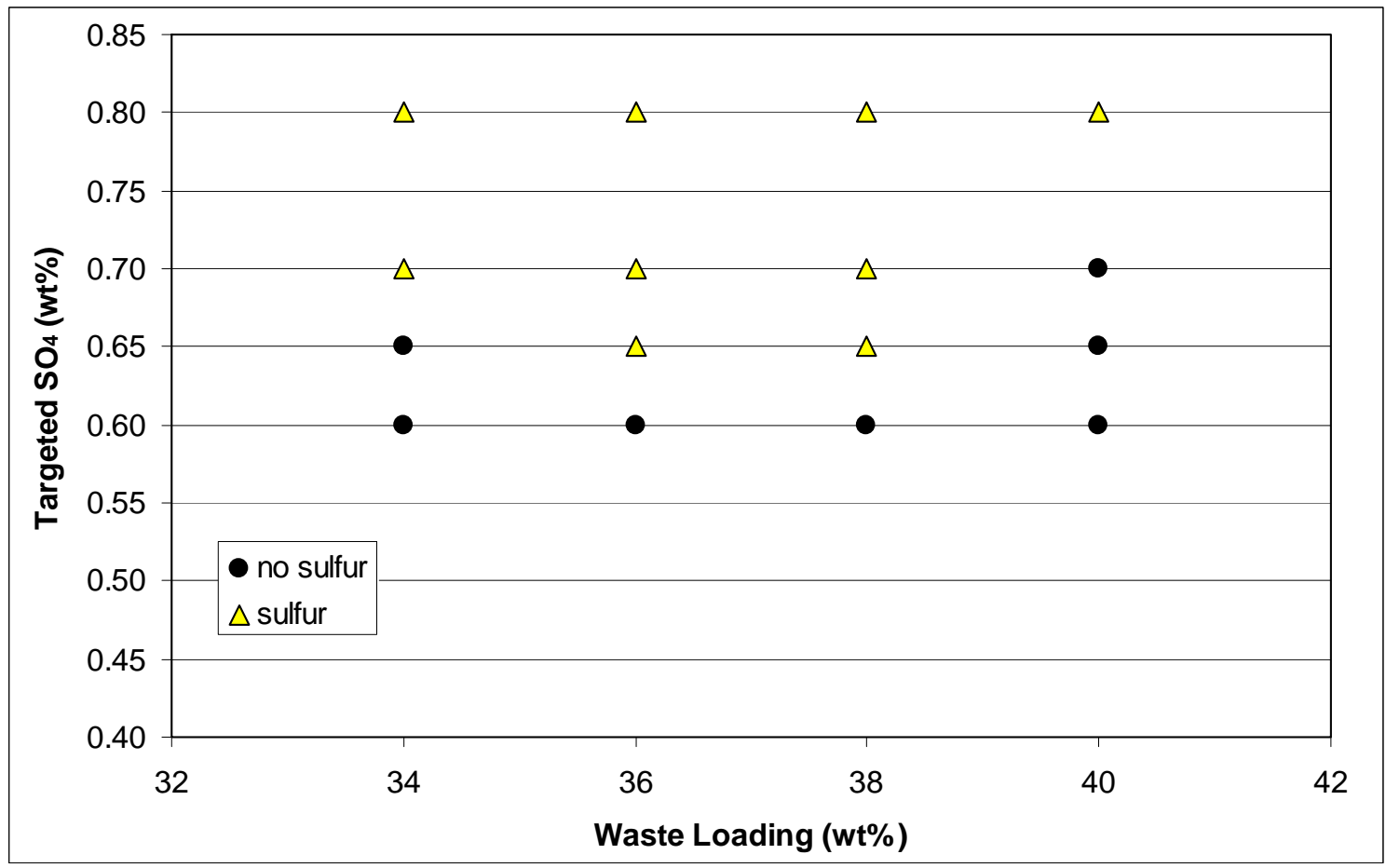

Figure 1. Visual observations for glasses based on Frit 418 and the SB7a-63" sludge composition as a function of waste loading and targeted $\mathrm{SO}_{4}{ }^{2-}$ content. 


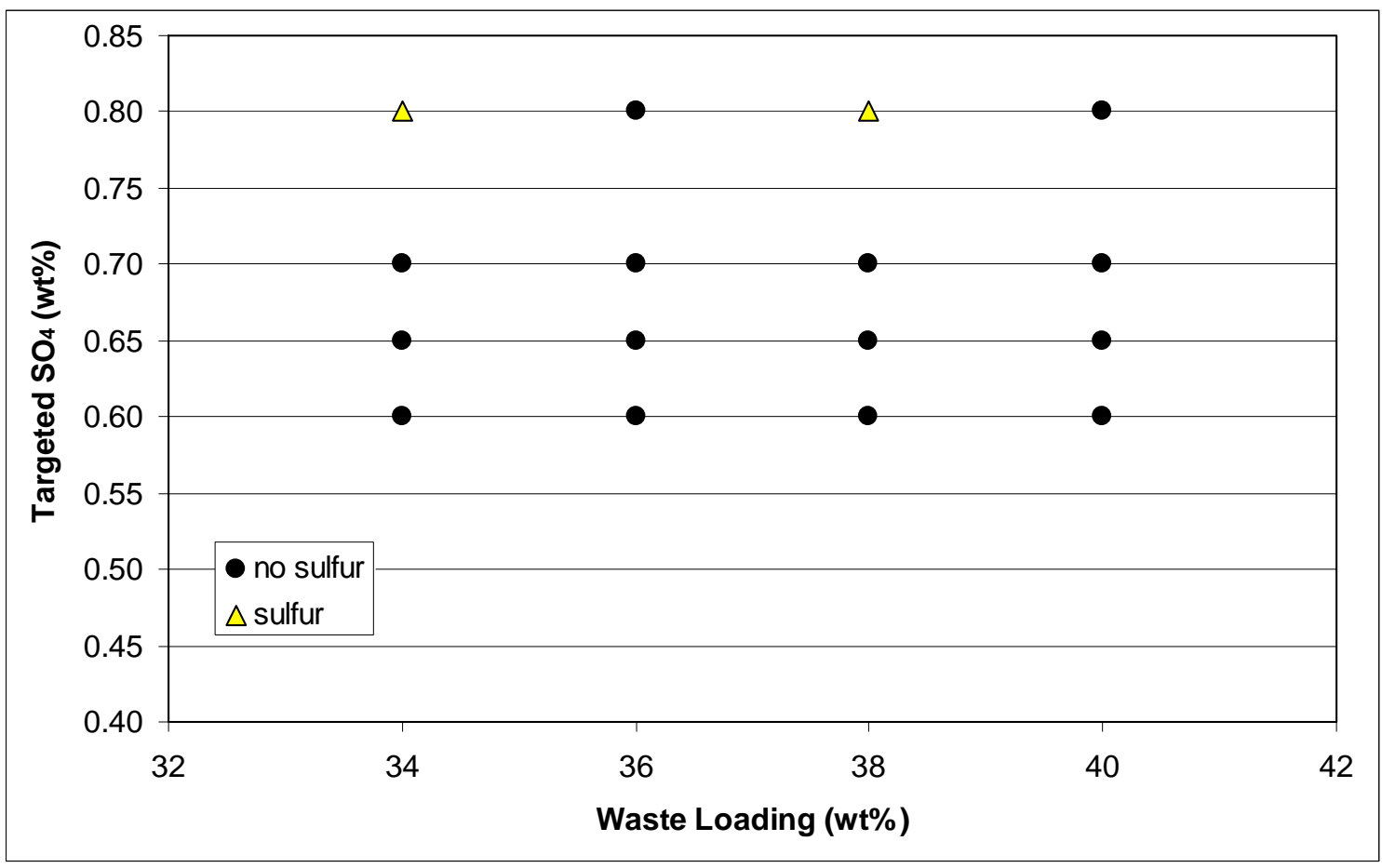

Figure 2. Visual observations for glasses based on Frit 418 and the SB7a-63"-ARP sludge composition as a function of waste loading and targeted $\mathrm{SO}_{4}{ }^{2-}$ content.

Chemical analysis of elemental S by ICP-AES was also conducted on all glasses fabricated in order to set an actual $\mathrm{SO}_{4}{ }^{2-}$ solubility limit for the Frit 418 and SB7a glass composition region for projected processing. The concentration of $\mathrm{SO}_{4}{ }^{2-}$ retained in each glass along with visual observations and the percent relative difference of the measured $\mathrm{SO}_{4}{ }^{2-}$ concentration from targeted are listed in Table 3-1. A reference glass (LRM) was also measured for S during chemical analysis in order to establish the ability to measure $S$ accurately in the study glasses. The LRM measurements were shown to be repeatable and were within the reported values ${ }^{12}$ (in $\mathrm{SO}_{3}$ ) and can be found in Table 3-2. The data reflect (in Table 3-1) and also as are shown in Figure 3 andFigure 4, that most compositions exhibited some loss of $\mathrm{SO}_{4}{ }^{2-}$ from what was targeted. This can be attributed to possible volatilization since the crucibles the glasses were melted in were only covered with a loose fitting lid.

Notice that for the Frit 418 and SB7a-63" glass system, the glasses which exhibited a sulfate layer at a $0.65 \mathrm{wt} \% \mathrm{SO}_{4}{ }^{2-}\left(36\right.$ and $38 \mathrm{wt} \% \mathrm{WL}$ ) retained 0.62 and $0.63 \mathrm{wt} \% \mathrm{SO}_{4}{ }^{2-}$ in glass. This is still higher than is expected to be processed during non-coupled processing. As was previously mentioned, none of the glasses for the Frit 418, SB7a-63" and SB7a-63"-ARP sludge cases exhibited a sulfate salt layer on the surface of the test melts at $0.6 \mathrm{wt} \% \mathrm{SO}_{4}{ }^{2-}$ over all waste loadings of interest (up to waste loadings of $40 \%$ ), so a $0.60 \mathrm{wt} \% \mathrm{SO}_{4}{ }^{2-}$ for SB7a processing would be the highest limit achievable. 
Table 3-1. Summary of Visual Observations and Measured $\mathrm{SO}_{4}{ }^{2-}$ for Frit 418 and SB7a-63” with and without ARP at Various $\mathrm{SO}_{4}{ }^{2-}$ Target Concentrations

\begin{tabular}{|c|c|c|c|c|c|c|c|}
\hline Glass ID & $\begin{array}{c}\text { Frit } \\
\text { ID }\end{array}$ & $\% \mathrm{WL}$ & Sludge Type & $\begin{array}{c}\text { Target Wt } \\
\mathrm{OSO}_{4}{ }^{2-}\end{array}$ & $\begin{array}{c}\text { Sulfur } \\
\text { Observed }\end{array}$ & $\begin{array}{c}\text { Measured } \\
\mathrm{Wt}^{2} \% \mathrm{SO}_{4}{ }^{2-}\end{array}$ & \% Diff. \\
\hline SL1-01 & 418 & 40 & SB7a-63" & 0.60 & No & 0.58 & -3 \\
\hline SL1-02 & 418 & 40 & SB7a-63" & 0.65 & No & 0.72 & 11 \\
\hline SL1-03 & 418 & 40 & SB7a-63" & 0.70 & No & 0.69 & -1 \\
\hline SL1-04 & 418 & 40 & SB7a-63" & 0.80 & Yes & 0.77 & -4 \\
\hline SL1-09 & 418 & 38 & SB7a-63" & 0.60 & No & 0.63 & 5 \\
\hline SL1-10 & 418 & 38 & SB7a-63" & 0.65 & Yes & 0.63 & -3 \\
\hline SL1-11 & 418 & 38 & SB7a-63" & 0.70 & Yes & 0.70 & 0 \\
\hline SL1-12 & 418 & 38 & SB7a-63" & 0.80 & Yes & 0.75 & -6 \\
\hline SL1-17 & 418 & 36 & SB7a-63" & 0.60 & No & 0.54 & -10 \\
\hline SL1-18 & 418 & 36 & SB7a-63" & 0.65 & Yes & 0.62 & -5 \\
\hline SL1-19 & 418 & 36 & SB7a-63" & 0.70 & Yes & 0.64 & -9 \\
\hline SL1-20 & 418 & 36 & SB7a-63" & 0.80 & Yes & 0.74 & -8 \\
\hline SL1-25 & 418 & 34 & SB7a-63" & 0.60 & No & 0.60 & 0 \\
\hline SL1-26 & 418 & 34 & SB7a-63" & 0.65 & No & 0.63 & -3 \\
\hline SL1-27 & 418 & 34 & SB7a-63" & 0.70 & Yes & 0.69 & -1 \\
\hline SL1-28 & 418 & 34 & SB7a-63" & 0.80 & Yes & 0.74 & -8 \\
\hline SL1-05 & 4418 & 40 & "SB7a-63"-ARP & 0.60 & No & 0.60 & 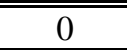 \\
\hline SL1-06 & 418 & 40 & SB7a-63"-ARP & 0.65 & No & 0.63 & -3 \\
\hline SL1-07 & 418 & 40 & SB7a-63"-ARP & 0.70 & No & 0.65 & -7 \\
\hline SL1-08 & 418 & 40 & SB7a-63"-ARP & 0.80 & No & 0.81 & 1 \\
\hline SL1-13 & 418 & 38 & SB7a-63"-ARP & 0.60 & No & 0.64 & 7 \\
\hline SL1-14 & 418 & 38 & SB7a-63"-ARP & 0.65 & No & 0.63 & -3 \\
\hline SL1-15 & 418 & 38 & SB7a-63"-ARP & 0.70 & No & 0.64 & -9 \\
\hline SL1-16 & 418 & 38 & SB7a-63"-ARP & 0.80 & Yes & 0.76 & -5 \\
\hline SL1-21 & 418 & 36 & SB7a-63"-ARP & 0.60 & No & 0.64 & 7 \\
\hline SL1-22 & 418 & 36 & SB7a-63"-ARP & 0.65 & No & 0.57 & -12 \\
\hline SL1-23 & 418 & 36 & SB7a-63"-ARP & 0.70 & No & 0.69 & -1 \\
\hline SL1-24 & 418 & 36 & SB7a-63"-ARP & 0.80 & No & 0.78 & -3 \\
\hline SL1-29 & 418 & 34 & SB7a-63"-ARP & 0.60 & No & 0.60 & 0 \\
\hline SL1-30 & 418 & 34 & SB7a-63"-ARP & 0.65 & No & 0.68 & 5 \\
\hline SL1-31 & 418 & 34 & SB7a-63"-ARP & 0.70 & No & 0.68 & -3 \\
\hline SL1-32 & 418 & 34 & SB7a-63"-ARP & 0.80 & Yes & 0.74 & -8 \\
\hline
\end{tabular}

Table 3-2. Measured $\mathrm{LRM} \mathrm{SO}_{3}$ Concentrations for Each Set of Glasses Submitted for Analytical Analysis

\begin{tabular}{||c|c||}
\hline LRM ID & Wt \% $\mathbf{S O}_{\mathbf{3}}$ \\
\hline LRM (A) & 0.23 \\
\hline LRM (B) & 0.23 \\
\hline LRM Target & 12 \\
\hline $\begin{array}{c}\text { LRM Measured } \\
\text { (Round Robin) }^{12}\end{array}$ & 0.2 \\
\hline
\end{tabular}




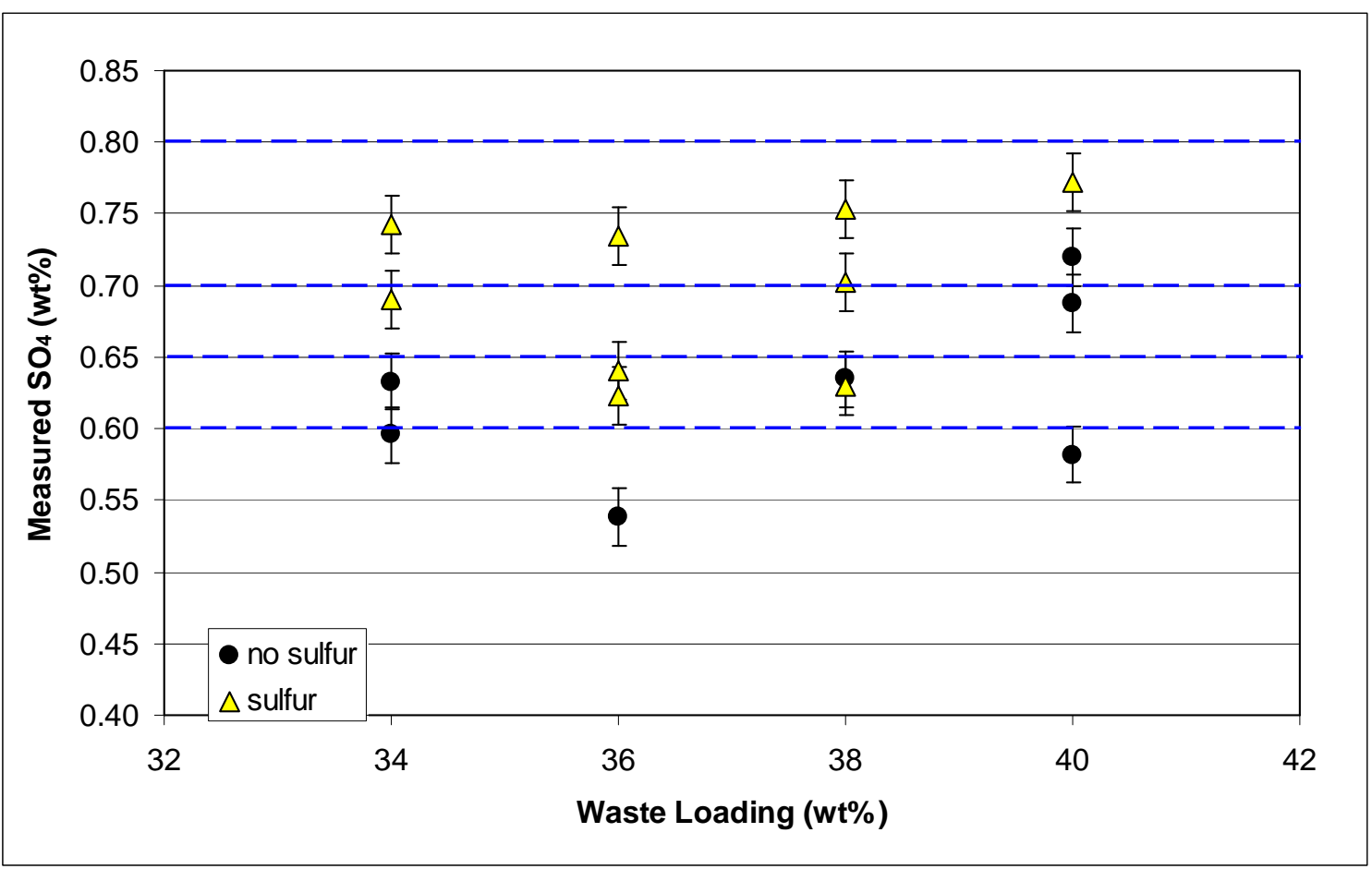

Figure 3. Visual observations for glasses based on Frit 418 and the SB7a-63" sludge composition as a function of waste loading and measured $\mathrm{SO}_{4}{ }^{2-}$ content.

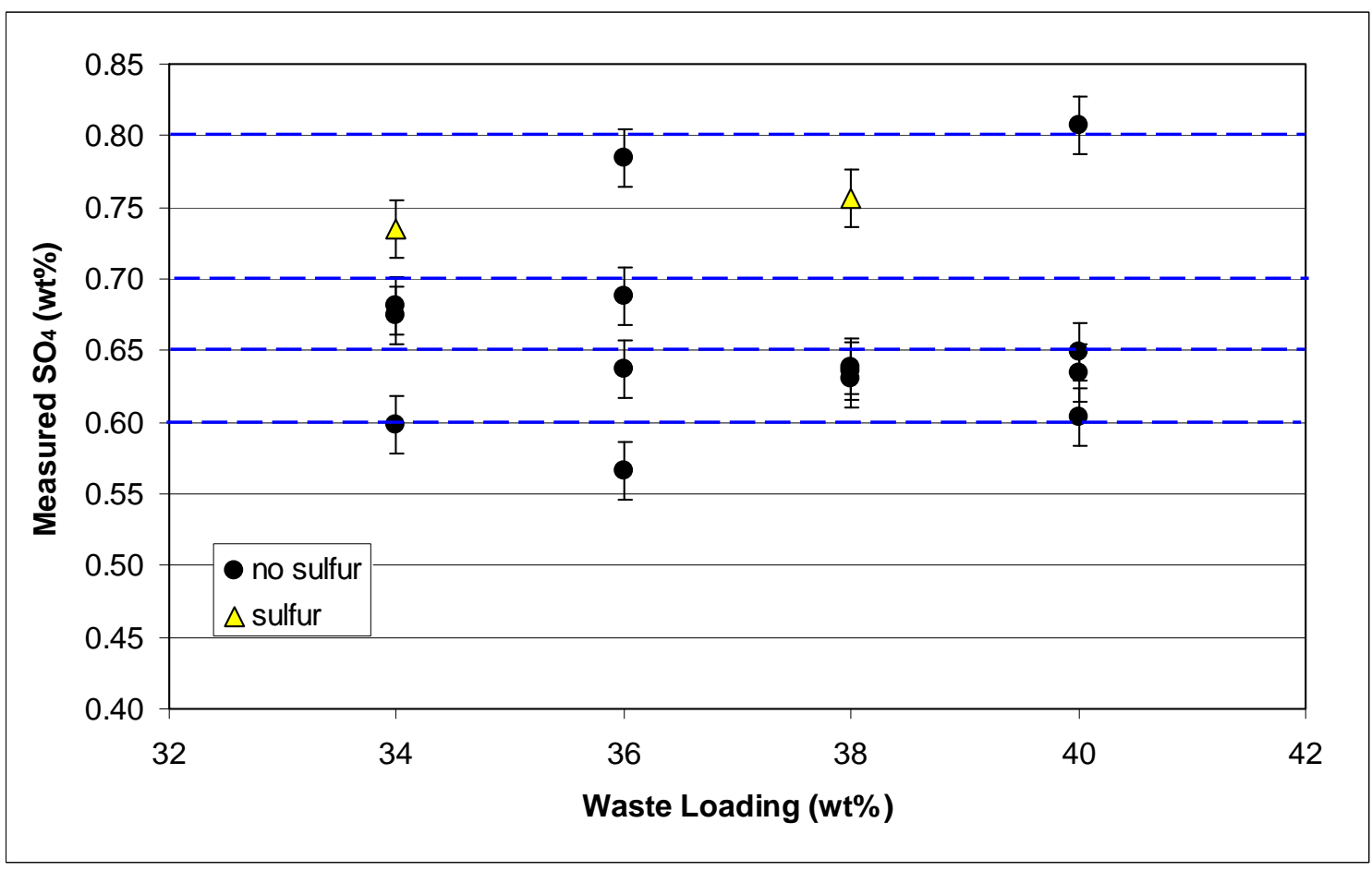

Figure 4. Visual observations for glasses based on Frit 418 and the SB7a-63"-ARP sludge composition as a function of waste loading and measured $\mathrm{SO}_{4}{ }^{2-}$ content. 
Another frit and sludge system was examined during the course of the study. Frit 702 was also recommended as a viable frit for SB7a processing ${ }^{7}$ and was used to evaluate whether the frit could achieve similar if not better $\mathrm{SO}_{4}{ }^{2-}$ solubility properties for $\mathrm{SB} 7 \mathrm{a}$ if projected concentrations of $\mathrm{SO}_{4}{ }^{2-}$ increased from those projected by SRR on 12-8-10. A smaller matrix of glasses was fabricated than what was conducted for the same sludge cases with Frit 418. Glasses with waste loadings of 34, 36, 38 and $40 \mathrm{wt} \%$ and $\mathrm{SO}_{4}{ }^{2-}$ concentrations of 0.7 and $0.8 \mathrm{wt} \%$ were fabricated for both SB7a-63" and SB7a-63"-ARP and exhibited the same visual results (shown in Figure 5). No visible sulfur layer was found on the surface of any of the glasses fabricated with Frit 702 even with the elevated concentrations of $\mathrm{SO}_{4}{ }^{2-}$ levels in glass.

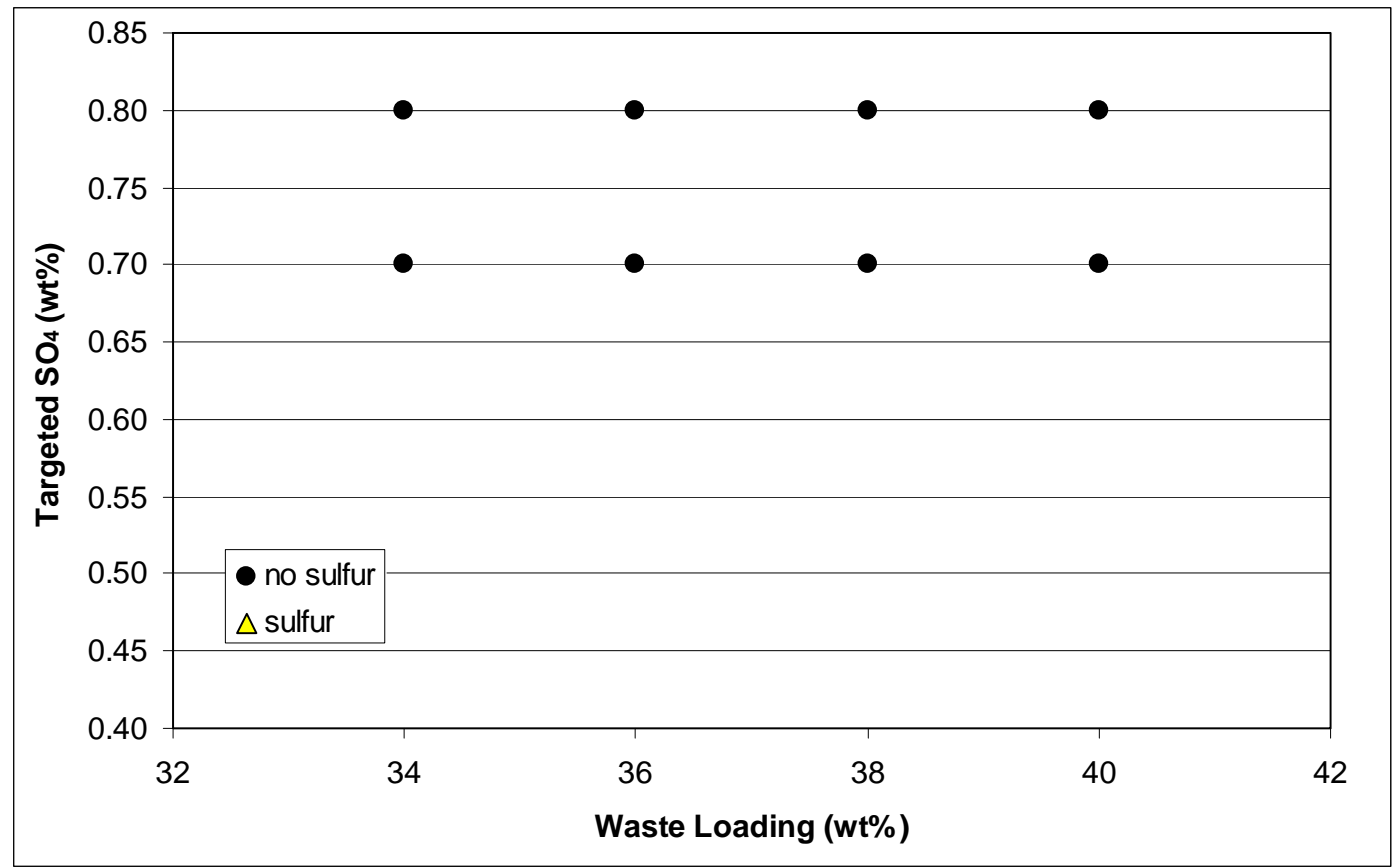

Figure 5. Visual observations for glasses based on Frit 702 and the SB7a-63" sludge case and the SB7a-63"-ARP sludge case as a function of waste loading and targeted $\mathrm{SO}_{4}{ }^{2-}$ content.

Chemical analysis of elemental S by ICP-AES was also conducted on all glasses fabricated in the Frit 702 system in order to evaluate the total retention of $\mathrm{SO}_{4}{ }^{2-}$ as compared to the Frit 418 system. The concentration of $\mathrm{SO}_{4}{ }^{2-}$ retained in each glass along with visual observations and \% relative difference of the measured $\mathrm{SO}_{4}{ }^{2-}$ concentration from targeted are listed in Table 3-3. There were no major differences evident in the percent differences from targeted to measured concentrations of $\mathrm{SO}_{4}{ }^{2-}$ between the two frits. Again, most glasses exhibited some loss of $\mathrm{SO}_{4}{ }^{2-}$ from what was targeted. This can also be attributed to possible volatilization since the crucibles the glasses were melted the same way, in crucibles with only a loose fitting lid. A visual plot of the measured $\mathrm{SO}_{4}{ }^{2-}$ concentration for the Frit 702 and SB7a-63" and SB7a-63"-ARP glasses are shown in Figure 6 and Figure 7. No apparent trend in retained $\mathrm{SO}_{4}{ }^{2-}$ as a function of waste loading was observed, but a number of glasses could retain as high as $0.79 \mathrm{wt} \% \mathrm{SO}_{4}{ }^{2-}$ in glass when 0.80 wt $\% \mathrm{SO}_{4}{ }^{2-}$ was targeted. From this small matrix study, it is highly probable that Frit 702 could increase the $\mathrm{SO}_{4}{ }^{2-}$ limit for the projected $\mathrm{SB} 7 \mathrm{a}$ waste processing higher than $0.60 \mathrm{wt} \% \mathrm{SO}_{4}{ }^{2-}$, which was found as the highest achievable limit for Frit 418. It is possible that the $\mathrm{SO}_{4}{ }^{2-}$ limit for the SB7a-Frit 702 projections could be as high as $0.80 \mathrm{wt} \% \mathrm{SO}_{4}{ }^{2-}$, but a small number of additional glasses would have to be fabricated to confirm the exact number since no glasses exhibited any visual evidence of sulfur on their surfaces. 
Table 3-3. Summary of Visual Observations and Measured $\mathrm{SO}_{4}{ }^{2-}$ for Frit 702 and SB7a-63" with and without ARP at Various $\mathrm{SO}_{4}{ }^{2-}$ Target Concentrations

\begin{tabular}{|c|c|c|c|c|c|c|c|}
\hline Glass ID & $\begin{array}{c}\text { Frit } \\
\text { ID }\end{array}$ & $\%$ WL & Sludge Type & $\begin{array}{c}\text { Target Wt } \\
\% \mathrm{SO}_{4}{ }^{2-} \\
\end{array}$ & $\begin{array}{c}\text { Sulfur } \\
\text { Observed }\end{array}$ & $\begin{array}{c}\text { Measured } \\
\mathrm{Wt}_{\% \mathrm{SO}_{4}{ }^{2-}}\end{array}$ & \% Diff. \\
\hline SL2-01 & 702 & 40 & SB7a-63" & 0.70 & No & 0.61 & -13 \\
\hline SL2-02 & 702 & 40 & SB7a-63" & 0.80 & No & 0.74 & -8 \\
\hline SL2-05 & 702 & 38 & SB7a-63" & 0.70 & No & 0.67 & -4 \\
\hline SL2-06 & 702 & 38 & SB7a-63" & 0.80 & No & 0.78 & -3 \\
\hline SL2-09 & 702 & 36 & SB7a-63" & 0.70 & No & 0.65 & -7 \\
\hline SL2-10 & 702 & 36 & SB7a-63" & 0.80 & No & 0.71 & -11 \\
\hline SL2-13 & 702 & 34 & SB7a-63" & 0.70 & No & 0.71 & 1 \\
\hline SL2-14 & 702 & 34 & SB7a-63" & 0.80 & No & 0.74 & -8 \\
\hline SL2-03 & 702 & 40 & $\begin{array}{l}\text { SB7a-63"-ARP } \\
\end{array}$ & 0.70 & No & 0.69 & $\begin{array}{l}-1 \\
\end{array}$ \\
\hline SL2-04 & 702 & 40 & SB7a-63"-ARP & 0.80 & No & 0.77 & -4 \\
\hline SL2-07 & 702 & 38 & SB7a-63"-ARP & 0.70 & No & 0.67 & -4 \\
\hline SL2-08 & 702 & 38 & SB7a-63"-ARP & 0.80 & No & 0.76 & -5 \\
\hline SL2-11 & 702 & 36 & SB7a-63"-ARP & 0.70 & No & 0.65 & -7 \\
\hline SL2-12 & 702 & 36 & SB7a-63"-ARP & 0.80 & No & 0.79 & -1 \\
\hline SL2-15 & 702 & 34 & SB7a-63"-ARP & 0.70 & No & 0.68 & -3 \\
\hline SL2-16 & 702 & 34 & SB7a-63"-ARP & 0.80 & No & 0.77 & -4 \\
\hline
\end{tabular}

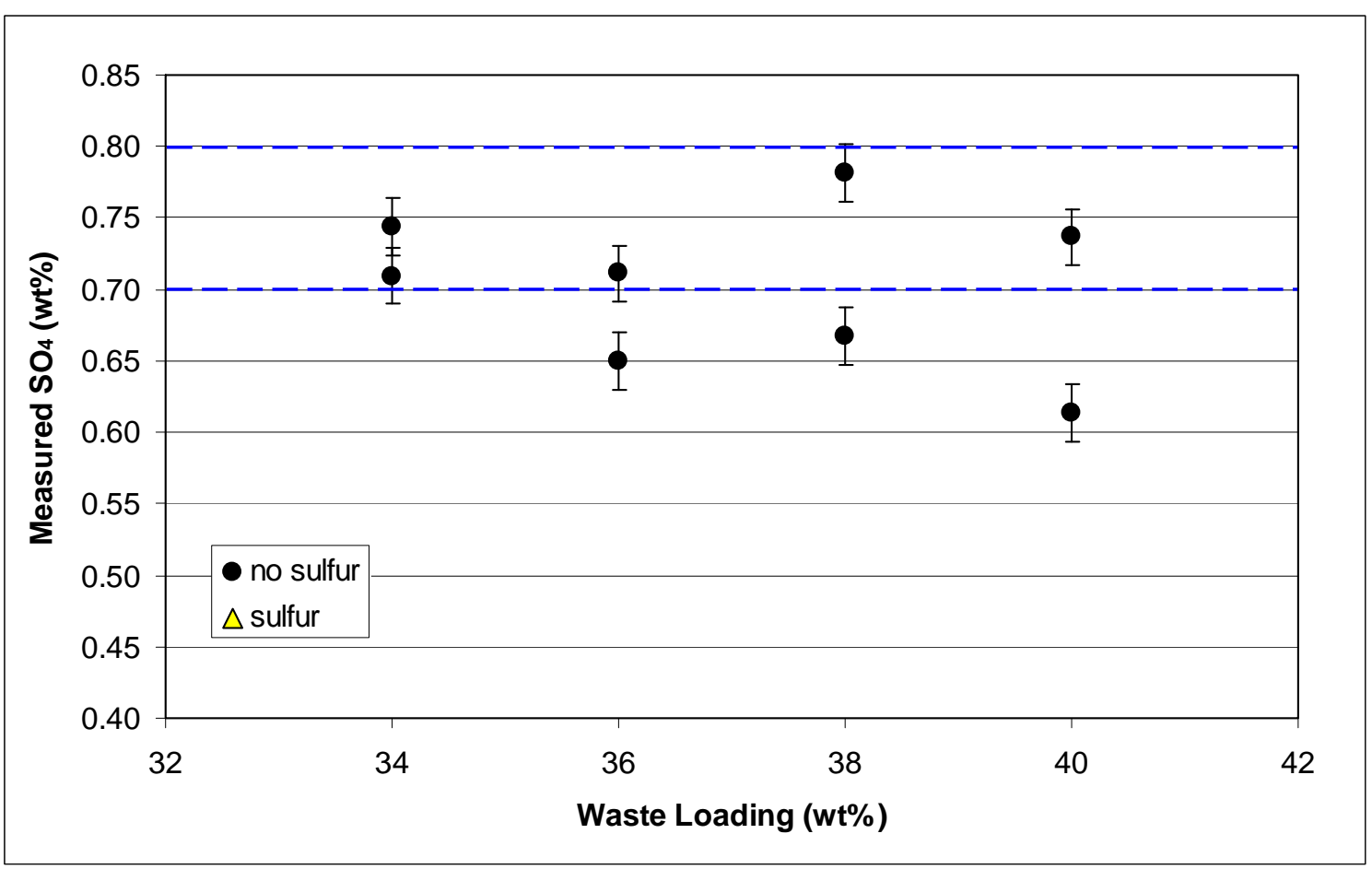

Figure 6. Visual observations for glasses based on Frit 702 and the SB7a-63" sludge composition as a function of waste loading and measured $\mathrm{SO}_{4}{ }^{2-}$ content. 


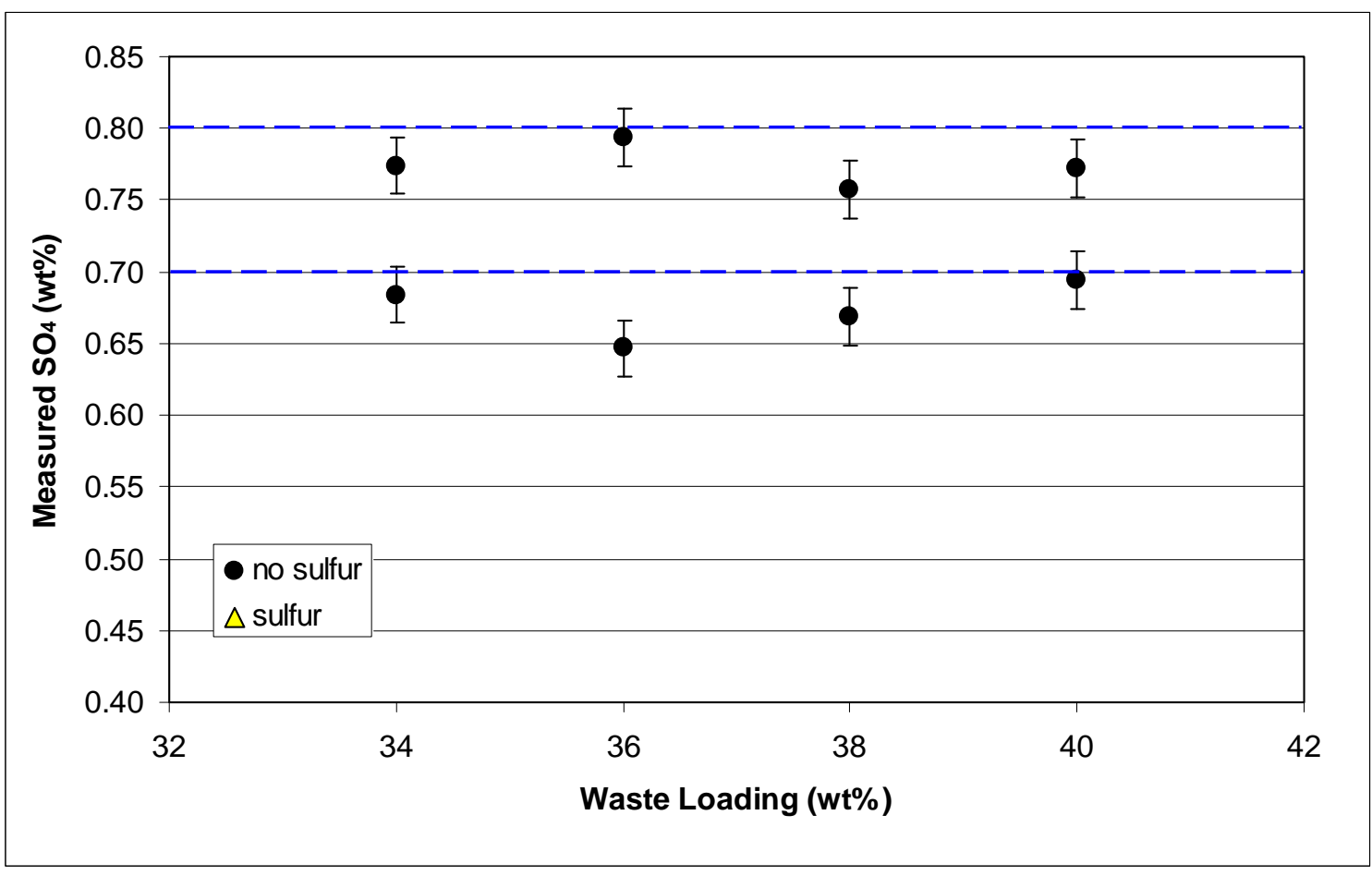

Figure 7. Visual observations for glasses based on Frit 702 and the SB7a-63"-ARP sludge composition as a function of waste loading and measured $\mathrm{SO}_{4}{ }^{2-}$ content.

\subsection{Conclusions}

Experiments conducted in this study examined $\mathrm{SO}_{4}{ }^{2-}$ retention and partitioning on a crucible scale as a function of composition from two sludge processing scenarios, two recommended frits and targeted operating windows for SB7a. A targeted $0.6 \mathrm{wt} \% \mathrm{SO}_{4}{ }^{2-}$ for waste loadings of $34 \%$ to $40 \%$ WL for Frit 418 and sludge cases SB7a-63" and SB7a-63"-ARP resulted in no partitioning of $\mathrm{SO}_{4}{ }^{2-}$ to a salt layer visible to the surface of the glass. When varying levels of $\mathrm{SO}_{4}{ }^{2-}$ were targeted for the Frit 418 and SB7a-63" and SB7a-63"-ARP sludge systems at waste loadings of $34 \%$ to $40 \%$, a strong correlation to sulfur partitioning and waste loading was not observed. The level of $\mathrm{SO}_{4}{ }^{2-}$ at which a visible sulfur layer was observed changed with slight composition differences (i.e., different waste loadings and the inclusion of the ARP stream).

The glasses with the lowest sulfate solubility limit, based on waste loading-sludge with Frit 418 were found at 36 and $38 \mathrm{wt} \% \mathrm{WL}$ for the sludge only case (SB7a-63”). While a targeted 0.6 wt $\% \mathrm{SO}_{4}{ }^{2-}$ concentration in glass resulted in no observed sulfur layer, at the slightly higher concentration of $0.65 \mathrm{wt} \% \mathrm{SO}_{4}{ }^{2-}$ both of these glasses exhibited sulfate on the surface and retained 0.62 and $0.63 \mathrm{wt} \% \mathrm{SO}_{4}{ }^{2-}$ in glass respectively. Due to the lower retention at the waste loadings of 36 and 38 wt \% WL with Frit 418 and the sludge only case (SB7a-63”), a 0.60 wt \% $\mathrm{SO}_{4}{ }^{2-}$ limit would be the limit for processing SB7a. A $0.60 \mathrm{wt} \% \mathrm{SO}_{4}{ }^{2-}$ limit would still be conservative as a feed limit for SB7a processing, since the current projected concentrations of $\mathrm{SO}_{4}{ }^{2-}$ for those particularly low solubility glasses would be 0.51 and $0.54 \mathrm{wt} \% \mathrm{SO}_{4}{ }^{2-}$ for SB7a63 ” and 0.54 and $0.57 \mathrm{wt} \% \mathrm{SO}_{4}{ }^{2-}$ for coupled operations (SB7a-63"-ARP) at the aforementioned waste loadings. Even more of a buffer is present since these projected concentrations of $\mathrm{SO}_{4}{ }^{2-}$ are on a non-radioactive basis that is, with $\mathrm{U}_{3} \mathrm{O}_{8}$ and $\mathrm{ThO}_{2}$ removed from the total sum of oxides, the 
total $\mathrm{SO}_{4}{ }^{2-}$ and the rest of the glass components are increased due to renormalization. It is also expected that the inclusion of the radioactive elements will not decrease the $\mathrm{SO}_{4}{ }^{2-}$ limit observed.

When Frit 702 was examined for sulfur solubility with the SB7a sludge projections, an increase in the $\mathrm{SO}_{4}{ }^{2-}$ limit from the Frit 418 system was observed. For either sludge case, spiking levels as high as $0.80 \mathrm{wt} \% \mathrm{SO}_{4}{ }^{2-}$ in glass resulted in no evidence of sulfur partitioning to the glass surface across the same waste loadings. These glasses, in some cases, achieved measured retention of $\mathrm{SO}_{4}{ }^{2-}$ as high as $0.79 \mathrm{wt} \%$ in glass, but saw no evidence that relative retention for any given combination of WL and spiking concentration was any different than when Frit 418 was used. If there is the potential that $\mathrm{SO}_{4}{ }^{2-}$ concentrations in SB7a are higher than projected and ultimately limit waste loadings given the $0.6 \mathrm{wt} \%$ limit determined with Frit 418, then Frit 702 is a viable option in order to increase the $\mathrm{SO}_{4}{ }^{2-}$ limit. Since the limit for SB7a and Frit 702 is above what was tested, a small number of additional glasses would have to be fabricated to confirm the highest $\mathrm{SO}_{4}{ }^{2-}$ limit achievable if Frit 702 becomes an option.

It is important to recognize that SRNL only examined two sludges and one frit scenario in great detail in order to determine how high of a $\mathrm{SO}_{4}{ }^{2-}$ limit could be obtained (specifically with Frit 418). This limit for both SB7a sludge cases with Frit 418 was 0.60 wt \% $\mathrm{SO}_{4}{ }^{2-}$. Any compositional variation or uncertainty in higher concentrations of $\mathrm{SO}_{4}^{2-}$ expected during processing could mean a change in the behavior of sulfur partitioning and a slightly different limit. From experimental results, a higher limit is expected with SB7a projections and Frit 702.

More uncertainty is involved when up-scaling to the DWPF melter and DWPF sludge processing. Vapor space conditions differ greatly in the large scale DWPF melter from those of a crucible in an electrically heated furnace. Varying REDOX state could also change the behavior of sulfur partitioning and result in a slightly different limit. In a previous study, ${ }^{6}$ fabricating glasses using feed material, frit and sealed crucible tests at the targeted $0.2 \mathrm{Fe}^{2+} / \Sigma \mathrm{Fe}$ REDOX value did not change the outcome of sulfate salt partitioning as compared to glasses fabricated using batched chemicals in covered crucibles. Ultimately, the actual amount of sulfate retained in the glass, and the amount that will volatilize or partition onto the surface will depend greatly upon the aforementioned conditions as well as various others. The experimental methods used to determine the $\mathrm{SO}_{4}{ }^{2-}$ concentration limit in glass for this study are conservative because they do not account for $\mathrm{SO}_{4}{ }^{2-}$ volatility known to occur in liquid feeding. Because not all of the melter conditions can be simulated on a laboratory scale accurately, ${ }^{13,14}$ the results from this study should be assessed as a stepping stone to evaluate $\mathrm{SO}_{4}{ }^{2-}$ retention in DWPF glass more in line with DWPF processing, in order to reduce some of the unknowns for future studies.

\subsection{Recommendations and Future Work}

It is recommended that, with Frit 418 expected to be used for SB7a processing and the received projections at 63" heel case in Tank 40, DWPF process SB7a with a limit of $0.6 \mathrm{wt} \% \mathrm{SO}_{4}{ }^{2-}$ in glass. If the final composition is very similar to the projected sludge composition from SRR on $12-08-10,{ }^{\mathrm{c}}$ a limit of $0.60 \mathrm{wt} \% \mathrm{SO}_{4}{ }^{2-}$ would be sufficient. Any increase in the projected concentration of $\mathrm{SO}_{4}{ }^{2-}$ would result in the possibility of exceeding the $\mathrm{SO}_{4}{ }^{2-}$ limit set with Frit 418 . One recommendation from SRNL is to change to Frit 702 for SB7a processing because of the higher sulfur tolerance observed in this study. If greater composition variation is seen prior to processing (heel level, higher expected waste loadings, change in washing endpoint, etc), or if a higher sulfate limit appears necessary, SRNL can evaluate the new projected region for the

\footnotetext{
c Sludge projections were received via personal communication from Daniel Mcilmoyle to David Peeler and Hasmukh Shah 12-08-2010.
} 
applicability of the $0.60 \mathrm{wt} \% \mathrm{SO}_{4}{ }^{2-}$ limit in glass or determine the upper $\mathrm{SO}_{4}{ }^{2-}$ limit in order to provide an additional recommendation.

It is also recommended that DWPF consider supporting further investigation of the dependence of $\mathrm{SO}_{4}{ }^{2-}$ retention related to glass composition. If enough of a correlation between $\mathrm{SO}_{4}{ }^{2-}$ retention and composition can be resolved, models could be developed and confirmed resulting in a less intense experimental need for $\mathrm{SO}_{4}{ }^{2-}$ limit determination for each sludge batch. To date, over 350 glass compositions have been generated and evaluated for elevated ( $>0.60 \mathrm{wt} \% \mathrm{SO}_{4}{ }^{2-}$ in glass) $\mathrm{SO}_{4}{ }^{2-}$ concentrations, however, most of these glasses targeted only two different sludge batches which makes them limited in their composition variation. A more "global" approach (similar to what SRNL has already started ${ }^{15}$ ) could lead to a more general limit which could apply to all future sludge batches. Also, since out-year processing targets higher waste loadings and the possible addition of sulfur laden waste streams, investigation of a frit additive in order to increase $\mathrm{SO}_{4}{ }^{2-}$ retention should warrant further investigation. Some success has been seen to date with additives such as $\mathrm{CaO}$ and $\mathrm{V}_{2} \mathrm{O}_{5}{ }^{16-18}$ Another approach would be further examining and modeling the DWPF melter conditions in order to calculate how much volatilization of $\mathrm{SO}_{4}{ }^{2-}$ is occurring during current and future processing conditions (effect of off-gas system, melter vapor space, implementation of bubblers, etc.). This approach could allow DWPF to assume some technical risk in implementing a higher limit with consideration that volatility will, in fact, occur. 


\subsection{References}

1. D.F. Bickford, A. Applewhite-Ramsey, C.M. Jantzen, and K. Brown, "Control of Radioactive Waste Glass Melters: I, Preliminary General Limits at Savannah River," J. Am. Ceram. Soc., 73 [10] 2896-902 (1990).

2. D.F. Bickford and C.M. Jantzen, "Inhibitor Limits for Washed Precipitate Based on Glass Quality and Solubility Limits," E.I. DuPont deNemours \& Co., Savannah River Company, Aiken, SC, U.S. Department of Energy DPST-86-546, 1986.

3. D.K. Peeler, C.C. Herman, M.E. Smith, T.H. Lorier, D.R. Best, T.B. Edwards, and M.A. Baich, "An Assessment of the Sulfate Solubility Limit for the Frit 418 - Sludge Batch 2/3 System," Westinghouse Savannah River Company, Aiken, SC, WSRC-TR-2004-00081, 2004.

4. K.M. Fox, T.B. Edwards, and D.K. Peeler, "Sulfate Retention in High Level Waste (HLW) Sludge Batch 4 (SB4) Glasses: A Preliminary Assessment," Washington Savannah River Company, Aiken, SC, WSRC-STI-2006-00038, 2006.

5. F.C. Raszewski, "An Assessment of the Applicability of the $0.6 \mathrm{Wt} \% \mathrm{SO}_{4}{ }^{2-}$ PCCS Limit for the Frit 418-SB5 System," Savannah River National Laboratory, Aiken, SC, Interoffice Memorandum, SRNL-L3100-2008-00023, 2008.

6. A.L. Billings, "DWPF Sulfate Limit Verification for SB6," Savannah River National Laboratory, Aiken, SC, SRNL-STI-2010-00191, 2010.

7. D.K. Peeler, "SB7a Frit Recommendation: Based on SB7a Compositional Projections from 12-8-10," Savannah River National Laboratory, Aiken, SC, Interoffice Memorandum, SRNL-L3100-2010-00248, 2010.

8. D.K. Peeler, "Task Technical and Quality Assurance Plan for Sludge Batch 7 - Impact of Sodium Oxalate and Sulfate on Glass," Savannah River National Laboratory, Aiken, SC, SRNL-RP-2010-00908, 2010.

9. $\quad$ S.G. Subosits, "Actinide Removal Process Material Balance Calculation with Low Curie Salt Feed," Savannah River Site, Aiken, SC, X-CLC-S-00113 Rev 0, Appendix J, September 24, 2004.

10. "Glass Melting," Savannah River National Laboratory, Aiken, SC, SRTC Procedure Manual, L29, ITS-0003, 2002. 
11. "Glass Batching," Savannah River National Laboratory, Aiken, SC, SRTC Procedure Manual, L29, ITS-0001, 2002.

12. W.L. Ebert and S.F. Wolf, "Round-Robin Testing of a Reference Glass for Low-Activity Waste Forms," Argonne National Laboratory, Chicago, IL, ANL-99/22, 1999.

13. C.M. Jantzen, M.E. Smith, and D.K. Peeler, "Dependency of Sulfate Solubility on Melt Composition and Melt Polymerization," Symposium on Waste Management Technologies in Ceramic and Nuclear Industries Proceedings. American Ceramic Society, United States, 2004.

14. C.M. Jantzen and M.E. Smith, "Revision of the Defense Waste Processing Facility (DWPF) Sulfate Solubility Limit," Savannah River National Laboratory, Aiken, SC, WSRC-TR-2003-00518, January, 2004.

15. A.L. Billings, "Sulfate Solubility Studies for DWPF Glasses," Savannah River National Laboratory, Aiken, SC, SRNL-RP-2009-00560, Rev. 1, 2009.

16. D.A. McKeown, I.S. Muller, K.S. Matlack, and I.L. Pegg, "X-ray Absorption Studies of Vanadium Valence and Local Environment in Borosilicate Waste Glasses Using Vanadium Sulfide, Silicate, and Oxide Standards," Journal of Non-Crystalline Solids, 298 [2-3] 160-75 (2002).

17. A.L. Youchak-Billings, "A Scoping Study Examining the Possibility of a Frit Additive to Increase Sulfur Solubility in DWPF Glass," Savannah River National Laboratory, Aiken, SC, Inter-office Memorandum, SRNL-PSE-2008-00173, 2008.

18. A.L. Billings and K.M. Fox, "Retention of Sulfate in Savannah River Site High-Level Radioactive Waste Glass," International Journal of Applied Glass Science, 1 [4] 388-400 (2010). 


\section{Distribution:}

J.W. Amoroso, 999-W

A.B. Barnes, 999-W

D.R. Best, 999-W

A.L. Billings, 999-W

J.M. Bricker, 704-27S

D.A. Crowley, 773-43A

T.B. Edwards, 999-W

H.H. Elder, 704-24S

T.L. Fellinger, 704-26S

S.D. Fink, 773-A

K.M. Fox, 999-W

J.M. Gillam, 766-H

C.C. Herman, 999-W

E.W. Holtzscheiter, 704-15S

J.F. Iaukea, 704-30S

P.R. Jackson, 703-46A

F.C. Johnson, 999-W

M.T. Keefer, 241-156H

P.A. Lee, 703-41A

D.W. Mcilmoyle, 766-H

S.L. Marra, 773-A

J.E. Occhipinti, 704-S

D.K. Peeler, 999-W

F.M. Pennebaker, 773-42A

H.M. Pittman, 704-27S

J.W. Ray, 704-S

A.R. Shafer, 704-27S

H.B. Shah, 766-H

D.C. Sherburne, 704-S

M.E. Smith, 704-30S

K.H. Subramanian, 766-H

A.V. Staub, 704-27S

M.E. Stone, 999-W 